\title{
Simulated Space Environmental Factors of Weightlessness, Noise and Low Air Pressure Differentially Affect the Circadian Rhythm and Gut Microbiome
}

\section{Huan Ma}

Sun Yat-sen University

Xihui Gan

Sun Yat-sen University

Jianwei Zhao

China Astronaut Research and Training Center

Yin Zhang

Sun Yat-sen University

Silin Li

Sun Yat-sen University

\section{Guanghan Kan}

China Astronaut Research and Training Center

\section{Bo Wang}

China Astronaut Research and Training Center

\section{Peng Zhang}

China Astronaut Research and Training Center

\section{Xiaohong Ma}

Sun Yat-Sen University Cancer Prevention and Treatment Center: Sun Yat-sen University Cancer Center Hongni Tian

National Institute of Biological Sciences

Meimei Liao

National Institute of Biological Sciences

Dapeng Ju

National Institute of Biological Sciences

Shuihong $\mathrm{Xu}$

China Astronaut Research and Training Center

\section{Lulin Chang}

Guangdong Jiangmen Chinese Traditional Medicine College

\section{Xiaoping Chen}

China Astronaut Research and Training Center 
Jinhu Guo ( $\nabla$ guojinhu@mail.sysu.edu.cn )

Sun Yat-Sen University https://orcid.org/0000-0003-4405-3959

\section{Research}

Keywords: Space, Circadian rhythm, Microgravity, Atmospheric pressure, Noise, Gut microbiome

Posted Date: April 5th, 2021

DOl: https://doi.org/10.21203/rs.3.rs-362076/v1

License: (c) (1) This work is licensed under a Creative Commons Attribution 4.0 International License. Read Full License 


\section{Abstract}

\section{Background}

he circadian clock extensively regulates physiology and behavior. In space, the astronauts encounter many environmental factors that are dramatically different from those on earth, however, the effects of these factors on circadian rhythms and the mechanisms remain largely unknown. The present study aimed to investigate the changes in the mouse circadian rhythm and gut microbiome under simulated space capsule conditions, including microgravity, noise and low atmospheric pressure.

Results

Noise and low atmospheric pressure were loaded in the capsule while the conditions in the animal room remained constant. The mice in the capsule showed disturbed locomotor rhythms and faster adaptation to a 6-h phase advance. RNA sequencing of hypothalamus samples revealed that microgravity simulated by hind limb unloading $(\mathrm{HU})$ and exposure to noise and low atmospheric pressure led to decreases in the quantities of differentially expressed genes (DEGs), including circadian clock genes. Changes in the rhythmicity of genes implicated in pathways of cardiovascular deconditioning and more concentrated circadian phases were found under $\mathrm{HU}$ or noise and low atmospheric pressure. Furthermore, 16S rRNA sequencing revealed dysbiosis in the gut microbiome, and noise and low atmospheric pressure may repress the temporal discrepancy in the microbiome community structure induced by microgravity. Changes in diel oscillation were observed in a number of gut bacteria with critical physiological consequences in metabolism and immunodefense.

\section{Conclusions}

Our data demonstrate that in addition to microgravity, exposure to noise and low atmospheric pressure affect the robustness of circadian rhythms and the community structure of the gut microbiome, and these factors may interfere with each other in their adaptation to respective conditions. These findings are important to further our understanding of the alteration of circadian rhythms in the space complex environment.

\section{Background}

Astronauts encounter environmental conditions that are dramatically different from those on the Earth's surface, including natural factors (gravity, radiation, magnetic field, lighting conditions, etc.) and social factors (shift work, confinement, emergency tasks, etc) [1-3]. In space, many astronauts' physiological and behavioral aspects are prone to be influenced by the environment, including fluid shift, body weight and nutrition, digesta propulsion and digestive function, cardiovascular functions, inflammation, transcriptional and metabolic changes, lipid level changes, microbiome response, and cognitive function [3-5]. 
It has also been demonstrated that the circadian rhythms of diverse organisms display significant changes in space [6-10]. Among different space environmental factors, the effects of microgravity and lighting conditions on circadian rhythms have been extensively investigated [9, 11-15]. However, the potential effects of other factors, such as noise and low atmospheric pressure, on circadian rhythms remain elusive.

In the manned space capsule, although the goal is to maintain a 1.0 atmospheric pressure (atm), the atmospheric pressure is usually lower, e.g., 0.9 atm, which is not considered to be unharmful to human health. During the Apollo missions, the environment inside the lunar module was $0.34 \mathrm{~atm}$ (5.0 psi) and $100 \%$ oxygen. It has been proposed that a lunar habitat will maintain an atmospheric pressure of 8-psi, which is approximately $0.54 \mathrm{~atm}[2,16]$. Brown et al proposed that diurnal and seasonal changes in atmospheric pressure play roles in modulating the metabolic rhythms of potato, and the rhythms of oxygen consumption in flatworm Dugesia [17]. In 2019, Kitahara et al revealed that higher hydrostatic pressure led to shortened circadian periods of the cyanobacteria circadian clock [18]. Low atmospheric pressure may cause disturbances in circadian rhythms due to decreased levels of dissolved oxygen in blood [19-21]. Hypoxia regulates the circadian clock by slowing the circadian cycle and dampening the amplitude through HIF1A, a key hypoxia-inducible factor. Low atmospheric pressure has been known to modulate circadian rhythms by slowing the circadian period and attenuating the amplitude due to hypoxia, which results in increased levels of HIF1A [19-20]. These findings suggest that hydrostatic or atmospheric pressure may affect circadian parameters.

The operation of various instruments and equipment on the space station results in environmental noise. According to a 2003 report, the average level of noise in the International Space Station was approximately $70 \mathrm{~dB}$ with a maximum of over $90 \mathrm{~dB}$ during sleep episodes [2]. Environmental noise, including noise in industrial settings may cause a broad range of physical consequences, including cardiac problems, sickness-related absenteeism, self-reported fatigue and disturbed sleep [22-24]. It has been reported that the night noise of road traffic noise affects blood glucose homeostasis due to disruption of circadian rhythms in diabetic patients but not nondiabetic patients [24]. However, the effects of noise on circadian rhythm and health remain to be further addressed.

The latest decade has seen rapid advances in the study of the gut microbiomes in different species. Gastrointestinal microorganisms play key regulatory roles in host physiological processes including metabolic immunity and neuroendocrine pathways [25-27]. In addition, accumulating evidence has demonstrated that host circadian rhythms modulate microbial oscillations, and vice versa $[28,29]$.

In space, the transcriptional and metabolic changes and microbiome responses between the twin astronauts are widely affected in the long-term space environment [4]. The changes in the gut microbiome of the mice were observed on a space shuttle mission (STS-135) for 13 days and after a 37-d spaceflight onboard the International Space Station $[5,30]$. However, the effects of space flight on the rhythms of the gut microbiome are unclear. 
The present study aimed to investigate the impacts of several simulated space environmental conditions, noise, low temperature and weight loss, on mouse circadian rhythms and the gut microbiome. The results demonstrate that these factors may impact circadian rhythms and the gut microbiome through modification of the circadian clock.

\section{Results}

\section{Combinational conditions of low air pressure and noise impact on locomotor rhythms}

In this study, 6 free-moving mice in the capsule and 6 free-moving mice in the animal room were raised for recording of locomotion rhytmicity. For hypothalamus and feces sampling, 3 mice were raised for each time point either in the capsule or animal room (Fig. 1a). Uniform field noise of $85 \mathrm{dbA}$ and $\sim 0.9$ atmospheric pressure were loaded in the simulated space capsule. We recorded the locomotion rhythms of control mice but not those of mice undergoing hind limb unloading $(\mathrm{HU})$ to mimic microgravity, in both the simulated space capsule and animal room. Compared to the mice in the animal room, the mice in the simulated space capsule showed a decrease in amplitude of locomotion during the days after the initiation of exposure to noise and low atmospheric pressure (Fig. 1b-d). Furthermore, it took the control mice in the capsule took $5.8 \pm 0.5 \mathrm{~d}$ to adjust to the 6-h advance of the LD cycles, which was significantly faster than that of the control mice in the animal room ( $7.7 \pm 0.8 \mathrm{~d}$ ) (Fig. 1b,c,e,f). These data suggest that noise and low atmospheric pressure may affect the function of the circadian system.

\section{Simulated space environmental factors differentially affect global gene expression}

To address the effects of different simulated space environmental factors (noise, low air pressure and microgravity) on global gene expression, the hypothalamus tissues containing suprachiasmatic nuclei $(\mathrm{SCN})$ from $\mathrm{HU}$ and control mice, in the capsule and animal room in the control (day 6) and experiment (day 36) periods, were collected for RNA sequencing (RNA-seq), respectively. Global changes in gene expression were observed (Fig. 2a,b). Notably, the quantities of differentially expressed genes (DEGs) in the capsule were much lower than those in the animal room (Fig. 2b,c). For instance, of the detected genes (Table S1), 22 were upregulated at the early stage and 151 were upregulated at the late stage of the control mice in the capsule while 884 were upregulated at the early stage and 4350 were upregulated at the late stage of the control mice in the animal room. Similarly, the number of DEGs was much higher in the HU mice (Fig. 2b-d; Figure S1b-g). It is worth noting that the number of DEGs in animals in SSC was much lower than those in AR, for both HU or control mice.

Gene enrichment and functional annotation by Kyoto Encyclopedia of Genes and Genomes (KEGG) pathway analysis indicated that a number of important biological processes associated with diseases were altered in the capsule mice, such as viral protein infection in $\mathrm{HU}$ mice and prion diseases in the 
control mice in the capsule. Pathways implicated in metabolism, neural systems, cell physiology, and diseases and cancers were also found. The pathway of circadian entrainment was enriched in the HU and control mice in the animal room (late vs. early) but not in the mice in the simulated space capsule. (Fig. 2e-f; Figure S1h-k; Table S3).

\section{Simulated space environmental factors differentially modify the rhythmicity of global gene expression}

JTK_Cycle analysis revealed differential reprogramming patterns of global circadian expression of hypothalamus transcripts between early and late stages under different conditions. For instance, 828 and 1558 genes of HU mice in the simulated space capsule showed rhythmicity in the early and late stages, respectively, and 78 genes showed rhymicity at both stages (Fig. 3a,b; Figure S2a-f; Table S2). Compared to $\mathrm{HU}$ mice in the animal room, the phase of global gene expression of $\mathrm{HU}$ mice in the capsule was limited in much narrower ranges at either early or late stages (Fig. 3b).

We next predicted the enriched pathways of those genes with significant changes in their rhythmicities under different conditions by KEGG pathway analysis. The pathways implicated in hypertrophic cardiomyopathy (HCM) and dilate cardiomyopathy (DCM) were identified between $\mathrm{HU}$ and control mice at the early stage in the animal room and between the early and late stages of HU mice in the animal room. Alterations in the rhythmicity of genes implicated in the pathway of circadian rhythm were identified between $\mathrm{HU}$ and control mice in the animal room at the late stage. Furthermore, the pathway associated with human papillomavirus infection was found in $\mathrm{HU}$ and noise and low atmospheric conditions. Additionally, pathways associated with cancers, cell physiology, immune defense, digestion, and neurodegenerative diseases were also found under the respective conditions (Fig. 3g-j; Figure S3; Table S4).

\section{Simulated space environmental factors differentially affect the expression of core circadian clock genes}

Together, the changes in locomotion rhythms (Fig. 1b-e) and pathways associated with circadian entrainment in the animal room (Fig. 3a-d) suggest that circadian rhythms may be affected at the molecular level. RNA-seq analysis confirmed that the average levels of circadian clock genes showed differentially altered gene expression patterns (Fig. 4a,b).

The average levels of many core circadian clock genes showed significant changes in mice in the animal room but not simulated space capsule (Fig. 4a-c). In the animal room, the levels of Clock, Per1, Per2, Per3, Cry1, Cry2, Nr1d1, Arntl and Npas2 were upregulated at the late stage in control mice, and Clock, Per2, Per3, Cry1, Cry2, Nr1d1 and Npas2 were upregulated in the HU mice. In contrast, none of these genes showed significant changes in the mice in the capsule. Arnt/2 showed no significant change in either HU or control mice (Fig. 4a,b). Relative levels of some circadian clock genes (late levels normalized to early levels), including Clock, Per1, Per2, Cry1, Cry2 and Nr1d1, were decreased in HU compared to control mice in the animal room. In contrast, the levels of these genes were comparable between $\mathrm{HU}$ and control mice 
in the simulated space capsule (Fig. 4c). Together, these data suggest that microgravity and noise and low atmospheric pressure may repress the expression and function of circadian clock genes. Changes in circadian rhythmicity (amplitude or phase) of these clock genes were also observed in most of these clock genes (Fig. 4d-k; Figure S4a-l).

The pressure was $0.9 \mathrm{~atm}$ in the capsule from day 7 to the end in the present study. The RNA-seq data showed no significant difference in the hypothalamus Hif1a level in control mice between the early and late stages in the capsule (Figure S4m,n). We further measured the HIF1A protein levels in liver samples, and revealed no significant change between these two stages; together, these data suggest that the low atmospheric pressure in the capsule is not sufficient to elicit hypoxia stress. However, a significant increase in HIF1A levels was observed in control mice at the late stage in the animal room and a significant decrease was observed in $\mathrm{HU}$ mice at the late stage in the capsule (Figure S40).

\section{Simulated space environmental factors differentially affect the abundance of the gut microbiome}

Stool samples from the transverse colon were subjected to 16S rRNA sequencing. In total, the sequenced microbiome consisted of 25 phyla, 58 classes, 82 orders, 129 families, and 172 genera of microorganisms, of which Bacteroidetes and Firmicutes were the most dominant. Together with Proteobacteria, Verrucomicrobia, Cyanobacteria, Actinobacteria, Deferribacteres, and Tenericutes, these phyla constituted over $99 \%$ of the known phylogenetic categories (Fig. 5a,b; Table S5).

The a-diversity between the data sets showed no significant difference (Fig. 5a-c). The PCoA results based on the OTU abundance revealed different separation under the indicated conditions (Figure S5a-h). Interestingly, a clear separation was visualized between the early and late stages of the HU mice gut microbiome in the animal room but not the HU mice in the simulated space capsule (Fig. 5d-g; Figure $\mathrm{S} 5 \mathrm{f}, \mathrm{h})$, suggesting that microgravity imposes long-term impacts on the structure of the gut microbial community, which could be repressed by superimposition with noise and low atmospheric pressure.

Among the detected phyla, changes in the abundance of many bacteria were observed (Fig. 6a-d; Figure S6). For instance, interestingly, Adlercreutzia was increased at the late stage of $\mathrm{HU}$ mice in the animal room but not in the capsule(Figure S6a,c). Verrucomicrobia abundance was increased the late stage of control mice in the capsule and the animal room but was decreased at the late stage of HU mice in the capsule and the animal room (Fig. 6a-d; Figure S6a-g).

PICRUSt analysis revealed that microbial pathways were significantly correlated with host cardiovascular functions. For instance, pathways of cardiac muscle contraction and HCM were identified between the early and late stages of control mice in both the animal room and the simulated space capsule, such as the renin-angiotensin system and HCM between the HU and control mice at the early stage in the simulated space capsule and cardiac muscle contraction, viral myocarditis, renin-angiotensin system and $\mathrm{HCM}$ pathways between the HU and control mice at the late stage in the simulated space capsule, and between $\mathrm{HU}$ and control mice at the late stage in the simulated space capsule. In addition, the pathway of 
amyotrophic lateral sclerosis was identified between the $\mathrm{HU}$ and control mice at the late stage in the simulated space capsule (Fig. 6e,f; Figure S7; Table S7). Other important pathways implicated in immune defense, metabolism, cancers, cell physiology, and other processes were also found under different conditions (Fig. 6e,f; Figure S7; Table S7).

\section{Simulated space environmental factors differentially affect the diel oscillation of gut microbiome}

Most of prokaryotic organisms possess no endogenous circadian systems; however, they exhibit diurnal rhythms owing to daily cycling environmental factors or the host internal milieu[31]. It has been revealed that mammalian oral and gut microbiomes undergo diurnal changes in the abundance of many microorganisms [32, 33].

The composition of some taxa displayed overt diurnal patterns over a day, including Akkermansia, Allobaculum, Bacteroides, Prevotella, Sutterella and so on (Fig. 7a). We next looked at the compositional changes of some bacteria at the genus level and found that the rhythmicities were changed under some conditions, such as Cetobacterium, Kaistobacter, Rhodoplanes, Acinetobacter, Corynebulcterium, Paraprevotella, Enterobacter, Devosia, etc (Fig. 7b-o; Figure S8; Table S6). Changes in phase or amplitude were found in some of these bacteria. For instance, Prevotella showed a higher amplitude in the HU mice in the capsule at the late stage than at the early, and a higher amplitude of Corynebacterium was seen in the control mice in the capsule. Rhodoplanes showed a higher amplitude in the HU mice in the capsule at the late stage. And Paraprevotella showed a dramatic increase in the oscillation in the HU mice at the late stage while it showed induction in abundance only at night in the control mice at the late stage in the animal room (Fig. 7b-o; Figure S8; Table S6).

\section{Discussion}

It is known that the space environment causes alterations in the circadian parameters of heart rate, body temperature, urinal variables, sleep-wake cycles and alertness $[1,13,14,34-37]$. Head-down bed rest in humans and hind limb unloading experiments in rodents are comparable ground-based approaches to mimic microgravity which leads to cephalad redistribution of body fluid which is similar to what occurs in space [38]. Head-down bed rest leads to changes in circadian/diel rhythms of physiological, metabolic and locomotion [39-43]. At the molecular level, upregulation of genes involved in circadian rhythm was found during and after 84 days of head-down tilt bed rest [12].

In space, the astronauts also encounter noise and low atmospheric pressure in the simulated space capsule in addition to microgravity; however, whether these two factors influence circadian rhythms remains elusive. In this work, we demonstrate that $\mathrm{HU}$ and the combination of noise and low atmospheric pressure differentially impact the expression of circadian clock genes and the rhythmicity of global gene expression. 
In mammals, circadian clock gene expression, architecture and consequent physiological and behavioral rhythms change along with development and aging [44]. The changes in clock gene expression between the early and late stages of mice in the animal room represent the development of molecular expression and the function of the circadian clock system in hypothalamus (Fig. 4). However, exposure to noise and low atmospheric pressure repressed the changes in global gene expression including those genes playing a role in the regulation of circadian rhythms. Moreover, we observed more concentrated phases of the oscillating genes of mice in the simulated space capsule. These data suggest that noise and low atmospheric pressure may hamper adaptation to microgravity at the transcriptomic level. The changed rhythms in locomotor and gene expression might be modulated through the master oscillator because the $\mathrm{SCN}$ resides in the hypothalamus [44].

Honda et al found that weight loss simulated by clinostate showed no effects on the expression of HIF1A in Kaposi's sarcoma-associated herpesvirus (KSHV)-infected cells [45]. Consistently, there was no significant change in Hif1a in mouse embryonic stem cells (mESCs) between spaceflight and ground control [46]. In contrast, the expression of HIF1A and HIF1-dependent transcripts in T lymphocytes and cells of the monocyte-macrophage system were changed in altered gravity simulated by parabolic flights [47]. Wang et al found that in a 28-d tail-suspension $\left(30^{\circ}\right)$, there was a significant increase in HIF1A in mouse hippocampus [48]. In this study we showed that $0.9 \mathrm{~atm}$ is not sufficient to cause hypoxia effects on circadian rhythms as the HIF1A level remained unchanged. We found significant increases in the control mice in the animal room for both Hif1a mRNA in hypothalamus and HIF1A protein in the liver and a significant increase in HIF1A protein in the liver in the control mice in the animal room, while Hif1a mRNA was not changed in hypothalamus. Together, these data suggest that HIF1A expression differs among tissues/cells, and more importantly, it is modulated by hypoxia-independent pathways.

Microgravity and its analogs cause comprehensive physiological changes including autonomic imbalances, cardiac atrophy, vascular impairment and microcirculatory dysfunction, which account for cardiovascular deconditioning [49-51]. In this work, we revealed that several pathways involving genes showing altered rhythmicities are associated with cardiovascular deconditioning (Fig. 3; Figure S3; Table S4), suggesting that rhythmicity is important for the genes associated with cardiovascular functions.

Noise is one of the globally prevalent environmental and occupational hazards, such as in cement plants and textile factories [52-54]. Accumulating evidence has demonstrated that dysbiosis of the gut microbiome is associated with the pathology of some cardiovascular disorders including atherosclerosis, hypertension and vascular dysfunction $[55,56]$. It has been reported that the diversity of the gut microbiome of White-Crowned Sparrow (Zonotrichia leucophrys) living in San Francisco Bay was altered upon exposure to environmental noise [57]. In this study, we showed that pathways associated with cardiovascular functions were enriched in many conditions including either $\mathrm{HU}$ or control and in either the simulated space capsule or the animal room (Fig. 6e,f; Figure S7; Table S7), suggesting that it was not specifically caused by HU or noise and low atmospheric pressure. However, it is worth noting that amyotrophic lateral sclerosis was specifically present between the early and late stages of HU mice in the simulated space capsule. 
Different gravitational conditions from $1 \mathrm{~g}$ on the surface of Earth and simulated microgravity have been shown to affect the gut microbiome in humans and rodents [5, 48, 58-61]. In this study we detected no significant change in a-diversity between all detected conditions (Fig. 5a). In contrast, significant changes in a-diversity have been reported in other studies under altered gravity or simulated microgravity conditions $[58,60]$. This inconsistency could be attributed to differences in sampling and calculation as we harvested the stool samples at six time points across a day, and the averaged values were used for adiversity calculation.

Changes in the abundance or rhythmicity of a number of bacteria were found in this work (Fig. 6; Fig. 7; Figure S8; Table S6). Among these microorganisms, Adlercreutzia can metabolize diadzein from soybean food to equol in the gut, which is considered to prevent from bone loss [62]. Therefore, the increase in gut Adlercreutzia at the late stage of HU mice in animal room suggests that it might be an adaptation to the HU condition, which might be repressed in by other factors like noise and low atmospheric pressure. A decrease in Prevotellaceae occurred in rats with uremia [63], Prevotella strains are associated with chronic inflammatory conditions including arthritis and HIV-1[64], and some Corynebacteria species are linked with a number of syndromes, such as granulomatous lymphadenitis, pneumonitis, pharyngitis, cutaneous infections and endocarditis [65]. Therefore, changes in rhythmicity may impose critical physiological effects under simulated microgravity, noise and low atmospheric pressure conditions. The changes in microbiome rhymicity are likely due to the modification of the mouse clock by environmental factors.

\section{Conclusions}

In this work we demonstrate that noise and low atmospheric pressure may also contribute to circadian disturbance and dysbiosis of the gut microbiome in space. In the future it will be critical to systematically study the effects of superimposition of these factors on the physiology, cognition and performance of astronauts. These findings will help further our understanding of complex space conditions on circadian rhythms, microbial alterations and their correlation with the physiology and health of the astronauts, and will improve the welfare of other professionals working in similar settings, e.g., environments with high levels of noise.

\section{Methods}

\section{Animals and bioethics}

Two-month-old C57BL/6N male mice were provided by Vital River Laboratories, Beijing. Half of these mice were raised in a simulated space capsule and the other half were raised in a normal animal room. Drinking water and food were provided by SPF Biotechnology Co., Ltd. (Beijing, China) and were provided ad libitum. 
There were six mice in the capsule and six in the animal room for locomotion monitoring. In both the capsule and the animal room, there were 36 mice undergoing $\mathrm{HU}$ and 36 free-moving mice as controls for sampling tissues along with some spare mice.

\section{Experimental conditions}

The simulated space capsule and the animal room are located in the Chinese Astronaut Research and Training Center. The simulated space capsule is a ground simulated platform of a simulated space capsule, in which temperature, humidity, air pressure and lighting conditions can be accurately controlled.

The light regime in the simulated capsule and animal room was a cycle alternating with $12 \mathrm{~h}$ of light and $12 \mathrm{~h}$ of dark (LD12:12). The illumination intensity of white light (full spectrum light) was approximately 200 lux. The conditions in the animal room were normal atmospheric pressure and weak noise 30-45 dBA throughout the experiment. In the simulated space capsule, the normal atmospheric pressure condition and weak noise (30-45 dBA) were set for days 1-6; a 0.9 atmospheric pressure environment (atm) and noise ( $85 \pm 2 \mathrm{dBA}$ ) were set for days 7-49 (Fig. 1a). On day 7 in the simulated space capsule, the $85 \mathrm{dbA}$ uniform field noise and $0.9 \mathrm{~atm}$ were added from 9:00 AM to the end of the experiment. The partial pressure of oxygen in the capsule after loading noise and low atmospheric pressure was $22 \pm 2$ $\mathrm{kPa}$, the partial pressure of carbon dioxide was $\ngtr 1 \mathrm{kPa}$ and the ammonia concentration was $\ngtr 20 \mathrm{ppm}$.

\section{Protocols and arrangements}

The hind limb unloading (HU) approach was employed to mimic the effects of weightlessness, which is a widely accepted ground-based weightlessness simulation [Ge et al 2019]. The device for hind limb unloading allows free movement throughout the cage with the body and the ground at $30^{\circ}$ [48].

The illumination time was 9:00 AM - 9:00 PM on days 1-37 and was adjusted to 3:00 AM - 3:00 PM on days 38-49 (Fig. 1a). Wheel running activity was monitored throughout the experiment and was analyzed with ClockLab Software (Actimetrics, Wilmette, USA).

The hypothalamus, liver and transverse colon containing stool of mice in both the capsule and the animal room were sampled at early and late stages, the early stage was from day 6 to day 7 (prior to the loading of noise and low atmospheric pressure in the capsule) and the late stage was from day 36 to day 37. The tissues were sampled every $4 \mathrm{~h}$ beginning at 9:00 AM and ending at 5:00 AM the next day. At each time point, three $\mathrm{HU}$ mice and three control mice were sacrificed and sampled.

\section{Monitoring and analysis of locomotor activity}

Six mice housed in the simulated space capsule and six mice in the animal room were living in mouse cages equipped with running wheels. Clocklab Analysis Software for Circadian Biology (Actimetrics Co. Ltd., USA) acquisition and analysis system collected the running wheel data of mice. The animal room data for days 38-39 were missing.

\section{RNA sequencing of the hypothalamus tissues}


The hypothalamic tissues were isolated and total RNA was extracted by using Trizol Reagent and subjected to RNA sequencing which was performed on a NovaSeq 6000 sequencer (Illumina, Co., Ltd., USA) by Forevergen Co. Ltd (Guangzhou, China). The reads length was PE150bp, and the qualified reads were obtained after removing raw reads with adapters or low quality bases, and then aligned to the mouse genome (NCBI37/MM9) by SOAP with default parameters. RSeQC was used to calculate the gene expression level represented as reads per kilobase per million mapped reads (RPKM) $[66,67]$. The JTK_CYCLE software was used to analyze and screen rhythm genes based on the RPKM values, and threshold for ADJ. $\mathrm{P} \leq 0.05$ and $\mathrm{PER} \geq 20 \mathrm{~h}$ and $\leq 24 \mathrm{~h}$ [68], and an analysis model with circadian repetition was used. To identify pathways with significant enrichment, pathway enrichment analysis was performed based on the KEGG database and the result of the analysis model with circadian repetition. KEGG pathways fulfilling the criterion of a hypergeometric $p<0.05 \& \log _{2}$ (FoldChange) $>1$ or $<-1$ were defined as significantly enriched in DEGs and were evaluated using DAVID (https://david.ncifcrf.gov/tools.jsp) to convert the ID to Entrez Gene ID for the species Mus musculus and the pathways were visualized using KOBAS (http://kobas.cbi.pku.edu.cn/kobas3/genelist/) [69].

\section{S rRNA sequencing and analysis}

The feces from the transverse colon lumen were harvested at fixed time points and genomic DNA was extracted per the manufacturer's protocol (QiaAmp DNA Stool Mini Kit). The DNA was then used as a template for polymerase chain reaction amplification of bacterial 16S rRNA genes. The primers 338F: $5^{\prime}-$ ACTCCTACGGGAGGCAGCA-3' and 806R: 5'- GGACTACHVGGGTWTCTAAT-3' were used to amplify the high-variation region (V3 V4) of $16 \mathrm{~S}$ rRNA. High-throughput sequencing was performed on an Illumina HiSeq 2500 in paired-ended mode by Forevergen Co. Ltd (Guangzhou, China).

The bioinformatics analysis of these sequences was used for OTU sequence clustering, clustering with operational taxonomic units (OUTs). OTU sequence clustering was performed with a consistency of $97 \%$. Clustering methods for upper bioinformatics analysis included OTU community and species analysis, beta diversity analysis, and significance analysis of species differences. Beta diversity on unweighted UniFrac was calculated by Quantitative Insights Into Microbial Ecology (QIIME) software (version 1.7.0; Novogene). Principal coordinate analysis (PCoA) was conducted to obtain principal coordinates and visualize from complex, multidimensional data. Unweighted Pair-Group Method with Arithmetic Euclidean distance was used to interpret the distance matrix using average linkage and was conducted by QIIME software (version 1.7.0; Novogene). LEfSe uses linear discriminant analysis (LDA) to estimate the effect of species abundance on the indicated conditions. KEGG functional profiles were predicted according to the taxonomic information with the phylogenetic information by reconstructing the whole microbial community, and Statistical Analysis of Metagenomic Profiles (STAMP) (v2.1.3, Welch's t-test, two-sided, post hoc comparisons (Benjamini-Hochberg FDR) $p<0.05$ ) $[70,71]$. The JTK_CYCLE software was used to analyze and screen rhythmicity of the microbiome, and threshold for ADJ. P $\leq 0.05$ and PER $\geq 20 \mathrm{~h}$ and $\leq 24 \mathrm{~h}[68]$.

\section{Western blot analysis of HIF1A protein in mouse liver}


Total proteins were extracted from mouse liver samples and subjected to SDS-polyacrylamide gel electrophoresis (7.5\%, containing a ratio of 37.5:1 acrylamide/bisacrylamide). Separated protein samples were transferred onto a polyvinylidene difluoride membrane with a micron pore size of $0.45 \mu \mathrm{m}$ (Merck, USA), followed by incubation with a primary antibody against mouse HIF1A (Lot. No. 42760, GeneTex, USA) and a horseradish peroxidase-conjugated goat-anti-rabbit secondary antibody (Bio-Rad, USA).

\section{Statistics}

One-way ANOVA was used to analyze the differences between groups in onset, the number of days with jet lag and hypothalamic clock gene relative levels in the simulated space capsule and the animal room. Student's $t$ test was used to analyze the different relative levels between groups in hypothalamic Hif1a and HIF1A of liver in the simulated space capsule and the animal room. Data in this experiment are expressed as mean (SE). ${ }^{\star} p<0.05,{ }^{*} p<0.01, \# p<0.001$.

\section{Abbreviations}

rRNA ribosomal RNA; HU:hind limb unloading; atm:atmosphere; HCM:hypertrophic cardiomyopathy; KEGG:Kyoto Encyclopedia of Genes and Genomes; LD12:12:12 h of light and $12 \mathrm{~h}$ of dark.

\section{Declarations}

\section{Authors' contributions}

J.G. and X.C. developed the project; H.M., X.G., J.Z., Y.Z., S.L., P.Z., H.T., M.L., D.J., L.C. and S.X. carried out experiments and collected data and samples. J.G., H.M., X.G., and X.M. analyzed the data. J.G. and X.G. wrote the manuscript with input from all of the authors. The author(s) read and approved the final manuscript.

\section{Acknowledgements}

We thank the staff in the animal center of China Astronaut Research and Training Center for their help and support, and thank Mr. Minghui He and Ms. Weifen Chen (Forevergen Co. Ltd, Guangzhou, China) for help in bioinformatics analysis.

\section{Funding}

This work was supported by the Open Fund of the National Key Laboratory of Human Factors Engineering in the Astronaut Center of China (SYFD180051809K) to J.G., the National Natural Science Foundation of China (No. 31871188) to J.G. and No. 32000824 to H.M, and the Space Medicine Research Program of China (HYZHXM03007) for J.G.

\section{Availability of data and materials}


The RNA-seq data sets are available at the GEO database under accession number GSE162309, and the raw 16S rRNA sequencing data have been deposited in in the NCBI Short Read Archive under project ID PRJNA679385.

\section{Ethics approval and consent to participate}

The protocol of mice experiments were approved by the China Astronaut Research and Training Center.

\section{Consent for publication}

Not applicable.

\section{Competing interests}

The authors declare no competing interests.

\section{Author details}

1 Ministry of Education (MOE) Key Laboratory of Gene Function and Regulation, School of Life Sciences, State Key Laboratory of Biocontrol, Sun Yat-sen University, Guangzhou 510006, China; 2State Key Laboratory of Space Medicine Fundamentals and Application, China Astronaut Research and Training Center, Beijing, 100094, China; 3National Institute of Biological Sciences, Beijing 102206, China. 4Guangdong Jiangmen Chinese Traditional Medicine College, Jiangmen 529000, China. 5National Key Laboratory of Human Factors Engineering, China Astronaut Research and Training Center, Beijing 100094, China.

\section{References}

1. Guo JH, Qu WM, Chen SG, Chen XP, Lv K, Huang ZL, et al. Keeping the right time in space: importance of circadian clock and sleep for physiology and performance of astronauts. Mil Med Res. 2014;1:23.

2. McPhee JC, Charles JB (eds). Human health and performance risks of space exploration missions: evidence reviewed by the NASA human research program. Houston, Texas: NASA Human Research Program; 2009.

3. Vernikos J. Human physiology in space. Bioessays. 1996;18(12):1029-37.

4. Garrett-Bakelman FE, Darshi M, Green SJ, Gur RC, Lin L, Macias BR, et al. The NASA Twins Study: A multidimensional analysis of a year-long human spaceflight. Science. 2019;364(6436): eaau8650.

5. Jiang P, Green SJ, Chlipala GE, Turek FW, Vitaterna MH. Reproducible changes in the gut microbiome suggest a shift in microbial and host metabolism during spaceflight. Microbiome. 2019;7(1):113.

6. Ma L, Ma J, Xu K. Effect of spaceflight on the circadian rhythm, lifespan and gene expression of Drosophila melanogaster. PLoS One. 2015;10(3):e0121600.

7. Mergenhagen $D$, Mergenhagen $E$. The biological clock of Chlamydomonas reinhardii in space. Eur $J$ Cell Biol. 1987;43(2):203-7. 
8. Hahn PM, Hoshizaki T, Adey WR. Circadian rhythms of the Macaca nemestrina monkey in Biosatellite 3. Aerosp Med. 1971;42(3):295-304.

9. Hoban-Higgins TM, Alpatov AM, Wassmer GT, Rietveld WJ, Fuller CA. Gravity and light effects on the circadian clock of a desert beetle, Trigonoscelis gigas. J Insect Physiol. 2003;49(7):671-5.

10. Sulzman FM, Ellman D, Fuller CA, Moore-Ede MC, Wassmer G. Neurospora circadian rhythms in space: a reexamination of the endogenous-exogenous question. Science. 1984; 225:232-4.

11. Alpatov AM. Studies of circadian rhythms in space flight: some results and prospects. Physiologist. 1991;34(1 Suppl):S145-6.

12. Fernandez-Gonzalo R, Tesch PA, Lundberg TR, Alkner BA, Rullman E, Gustafsson T. Three months of bed rest induce a residual transcriptomic signature resilient to resistance exercise countermeasures. FASEB J. 2020;34(6):7958-69.

13. Fuller CA, Hoban-Higgins TM, Griffin DW, Murakami DM. Influence of gravity on the circadian timing system. Adv Space Res. 1994;14(8):399-408.

14. Fuller CA. The effects of gravity on the circadian timing system. J Gravit Physiol. 1994;1(1):P1-4.

15. Wang D, Zhang L, Liang X, Shen H, Chen X, Wan Y, et al. Space Meets Time: Impact of Gravity on Circadian/Diurnal Rhythms. A sponsored supplement to Science: Human performance in space advancing astronautics research in China. 2014;15-17.

16. McDonnell AC, Eiken O, Mekjavic PJ, Mekjavic IB. Circadian rhythm of peripheral perfusion during 10day hypoxic confinement and bed rest. Eur J Appl Physiol. 2014;114(10):2093-104.

17. Brown FA Jr, Hastings JW, Palmer JD. The Biological Clock. Two Views. New York: Academic Press; 1970. P.48-56.

18. Kitahara R, Oyama K, Kawamura T, Mitsuhashi K, Kitazawa S, Yasunaga K, et al. Pressure accelerates the circadian clock of cyanobacteria. Sci Rep. 2019;9(1):12395.

19. Adamovich Y, Ladeuix B, Golik M, Koeners MP, Asher G. Rhythmic Oxygen Levels Reset Circadian Clocks through HIF1alpha. Cell Metab. 2017;25(1):93-101.

20. Peek CB, Levine DC, Cedernaes J, Taguchi A, Kobayashi Y, Tsai SJ, et al. Circadian Clock Interaction with HIF1alpha Mediates Oxygenic Metabolism and Anaerobic Glycolysis in Skeletal Muscle. Cell Metab. 2017;25(1):86-92.

21. Wu Y, Tang D, Liu N, Xiong W, Huang H, Li Y, et al. Reciprocal regulation between the circadian clock and hypoxia signaling at the genome level in mammals. Cell Metab. 2017;25(1):73-85.

22. Halperin D. Environmental noise and sleep disturbances: A threat to health? Sleep Sci. 2014;7(4):209-12.

23. Leather P, Beatle D, Sullivan L. Noise, psychosocial stress and their interaction in the workplace. J Environ Pschol. 2003;23(2):213-222.

24. Eze IC, Imboden M, Foraster M, Schaffner E, Kumar A, Vienneau D, et al. Exposure to Night-Time Traffic Noise, Melatonin-Regulating Gene Variants and Change in Glycemia in Adults. Int J Environ Res Public Health. 2017;14(12):1492. 
25. Farzi A, Fröhlich EE, Holzer P. Gut Microbiota and the Neuroendocrine System. Neurotherapeutics. 2018;15(1):5-22.

26. Van Treuren W, Dodd D. Microbial Contribution to the Human Metabolome: Implications for Health and Disease. Annu Rev Pathol. 2020;15:345-69.

27. Sittipo P, Lobionda S, Lee YK, Maynard CL. Intestinal microbiota and the immune system in metabolic diseases. J Microbiol. 2018;56(3):154-162.

28. Bishehsari F, Voigt RM, Keshavarzian A. Circadian rhythms and the gut microbiota: from the metabolic syndrome to cancer. Nat Rev Endocrinol. 2020;16(12):731-39.

29. Pearson JA, Wong FS, Wen L. Cross talk between circadian rhythms and the microbiota. Immunology. 2020;161(4):278-90.

30. Ritchie LE, Taddeo SS, Weeks BR, Lima F, Bloomfield SA, Azcarate-Peril MA, et al. Space Environmental Factor Impacts upon Murine Colon Microbiota and Mucosal Homeostasis. PLoS One. 2015;10(6):e0125792.

31. Kelly LW, Nelson CE, Haas AF, Naliboff DS, Calhoun S, Carlson CA, et al. Diel population and functional synchrony of microbial communities on coral reefs. Nat Commun. 2019;2;10(1):1691.

32. Collado MC, Engen PA, Band'ın C, Cabrera-Rubio R, Voigt RM, Green SJ, et al. Timing of food intake impacts daily rhythms of human salivary microbiota: a randomized, crossover study. FASEB J. 2018(4);32:2060-72.

33. Thaiss CA, Levy M, Korem T, Dohnalová L, Shapiro H, Jaitin DA, et al. Microbiota Diurnal Rhythmicity Programs Host Transcriptome Oscillations. Cell. 2016;167(6):1495-1510.e12.

34. Flynn-Evans EE, Barger LK, Kubey AA, Sullivan JP, Czeisler CA. Circadian misalignment affects sleep and medication use before and during spaceflight. NPJ Microgravity. 2016; 2:15019.

35. Fujita SI, Rutter L, Ong Q, Muratani M. Integrated RNA-seq Analysis Indicates Asynchrony in Clock Genes between Tissues under Spaceflight. Life. 2020;10(9):196.

36. Liu Z, Luo G, Du R, Sun W, Li J, Lan H, et al. Effects of spaceflight on the composition and function of the human gut microbiota. Gut Microbes. 2020;11(4):807-19.

37. Stampi C. Sleep and circadian rhythms in space. J Clin Pharmacol. 1994;34(5):518-34.

38. Morey-Holton ER, Globus RK. Hindlimb unloading rodent model: technical aspects. J Appl Physiol. 2002;92(4):1367-77.

39. Liang X, Zhang L, Shen H, Chen X, Wan Y, Li L, et al. Effects of a 45-day head-down bed rest on the diurnal rhythms of activity, sleep and heart rate. Biol Rhythm Res. 2014;45(4):596-601.

40. Liang X, Zhang L, Wan Y, Yu X, Guo Y, Chen X, et al. Changes in the Diurnal Rhythms during a 45-Day Head-Down Bed Rest. PLoS One. 2012;7(10): e47984.

41. Mendt S, Maggioni MA, Nordine M, Steinach M, Opatz O, Belavý D, et al. Circadian rhythms in bed rest: Monitoring core body temperature via heat-flux approach is superior to skin surface temperature. Chronobiol Int. 2017;34(5):666-76. 
42. Morris CJ, Hastings JA, Boyd K, Krainski F, Perhonen MA, Scheer FA, et al. Day/night variability in blood pressure: influence of posture and physical activity. Am J Hypertens. 2013;26(6):822-8.

43. Solbiati S, Landreani F, Turcato M, Martin-Yebra A, Costantini L, Vaida P, et al. Analysis of changes in cardiac circadian rhythms of RR and QT induced by a 60-day head-down bed rest with and without nutritional countermeasure. Eur J Appl Physiol. 2020;120(7):1699-710.

44. Hood S, Amir S. The aging clock: circadian rhythms and later life. J Clin Invest. 2017;127(2):437-446.

45. Honda T, Nakayama R, Kawahara Y, Yuge L, Ueda K. Kaposi's sarcoma-associated herpesvirus is cellintrinsically controlled in latency in microgravity. Virus Res. 2020; 276:197821.

46. Blaber EA, Finkelstein H, Dvorochkin N, Sato KY, Yousuf R, Burns BP, et al. Microgravity Reduces the Diffrentiation and Regenerative Potential of Embryonic Stem Cells. Stem Cells Dev. 2015;24(22):2605-21.

47. Vogel J, Thiel CS, Tauber S, Stockmann C, Gassmann M, Ullrich O. Expression of Hypoxia-Inducible Factor 1alpha (HIF-1alpha) and Genes of Related Pathways in Altered Gravity. Int J Mol Sci. 2019;20(2):436.

48. Wang T, Chen H, Lv K, Ji G, Liang F, Zhang Y, et al. Activation of HIF-1a and its downstream targets in rat hippocampus after long-term simulated microgravity exposure. Biochem Biophys Res Commun. 2017 Apr 8;485(3):591-597.

49. Malkani S, Chin CR, Cekanaviciute E, Mortreux M, Okinula H, Tarbier M, et al. Circulating miRNA Spaceflight Signature Reveals Targets for Countermeasure Development. Cell Rep. 2020;108448.

50. Moffitt JA, Henry MK, Welliver KC, Jepson AJ, Garnett ER. Hindlimb unloading results in increased predisposition to cardiac arrhythmias and alters left ventricular connexin 43 expression. Am J Physiol Regul Integr Comp Physiol. 2013;304(5): R362-73.

51. Navasiolava N, Yuan M, Murphy R, Robin A, Coupé M, Wang L, et al. Vascular and Microvascular Dysfunction Induced by Microgravity and Its Analogs in Humans: Mechanisms and Countermeasures. Front Physiol. 2020; 11:952.

52. Basner M, Brink M, Bristow A, de Kluizenaar Y, Finegold L, Hong J, et al. ICBEN review of research on the biological effects of noise 2011-2014. Noise Health. 2015;17(75):57-82.

53. Tak S, Davis RR, Calvert GM. Exposure to hazardous workplace noise and use of hearing protection devices among US workers--NHANES, 1999-2004. Am J Ind Med. 2009;52(5):358-71.

54. Zhang C, Yuan S, Li D. Comprehensive Control of the Noise Occupational Hazard in Cement Plant. Procedia Eng. 2012; 43:186-90.

55. Battson ML, Lee DM, Weir TL, Gentile CL. The gut microbiota as a novel regulator of cardiovascular function and disease. J Nutr Biochem. 2018; 56:1-15.

56. Guzik TJ, Channon KM. Linking noise to cardiovascular disease pathogenesis. Eur Heart J. 2017;38(37):2850-2852.

57. Berlow M, Phillips JN, Derryberry EP. Effects of Urbanization and Landscape on Gut Microbiomes in White-Crowned Sparrows. Microb Ecol. 2020; 81: 253-266. 
58. Alauzet C, Cunat L, Wack M, Lozniewski A, Busby H, Agrinier N, et al. Hypergravity disrupts murine intestinal microbiota. Sci Rep. 2019;9(1):9410.

59. Li P, Shi J, Zhang P, Wang K, Li J, Liu H, et al. Simulated microgravity disrupts intestinal homeostasis and increases colitis susceptibility. FASEB J. 2015;29(8):3263-73.

60. Shi J, Wang Y, He J, Li P, Jin R, Wang K, et al. Intestinal microbiota contributes to colonic epithelial changes in simulated microgravity mouse model. FASEB J. 2017;31(8):3695-709.

61. Voorhies AA, Mark Ott C, Mehta S, Pierson DL, Crucian BE, Feiveson A, et al. Study of the impact of long-duration space missions at the International Space Station on the astronaut microbiome. Sci Rep. 2019;9(1):9911.

62. Nitert MD, Mousa A, Barrett HL, Naderpoor N, de Courten B. Altered Gut Microbiota Composition Is Associated With Back Pain in Overweight and Obese Individuals. Front Endocrinol. 2020;11:605.

63. Vaziri ND, Wong J, Pahl M, Piceno YM, Yuan J, DeSantis TZ, et al. Chronic kidney disease alters intestinal microbial flora. Kidney Int. 2013;83(2):308-15.

64. Ley RE. Gut microbiota in 2015: Prevotella in the gut: choose carefully. Nat Rev Gastroenterol Hepatol. 2016;13(2):69-70.

65. Lipsky BA, Goldberger AC, Tompkins LS, Plorde JJ. Infections caused by nondiphtheria corynebacteria. Rev Infect Dis. 1982;4(6):1220-35.

66. Li R, Yu C, Li R, Lam TW, Yiu SM, Kristiansen K, et al. SOAP2: an improved ultrafast tool for short read alignment. Bioinformatics. 2009;25(15):1966-7.

67. Wang L, Wang S, Li W. RSeQC: quality control of RNA-seq experiments. Bioinformatics. 2012;28(16):2184-5.

68. Hughes ME, Hogenesch JB, Kornacker K. JTK_CYCLE: an efficient nonparametric algorithm for detecting rhythmic components in genome-scale data sets. J Biol Rhythms. 2010;25(5):372-80.

69. Xie C, Mao X, Huang J, Ding Y, Wu J, Dong S, et al. KOBAS 2.0: a web server for annotation and identification of enriched pathways and diseases. Nucleic Acids Res. 2011;39(Web Server issue):W316-22.

70. Langille MGI, Zaneveld J, Caporaso J, McDonald D, Knights D, Reyes JA, et al. Predictive functional profiling of microbial communities using 16S rRNA marker gene sequences. Nat. Biotechnol. 2013;31(9): 814-21.

71. Parks DH, Tyson GW, Hugenholtz P, Beiko RG. STAMP: Statistical analysis of taxonomic and functional profiles. Bioinformatics 2014;30(21): 3123-4.

\section{Figures}


a

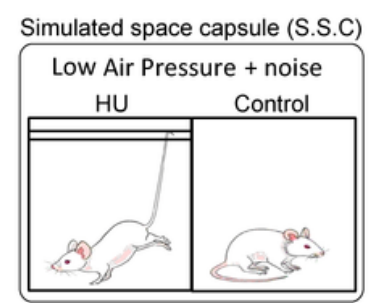

ZT (h) $0246 \quad 81012141618202224$

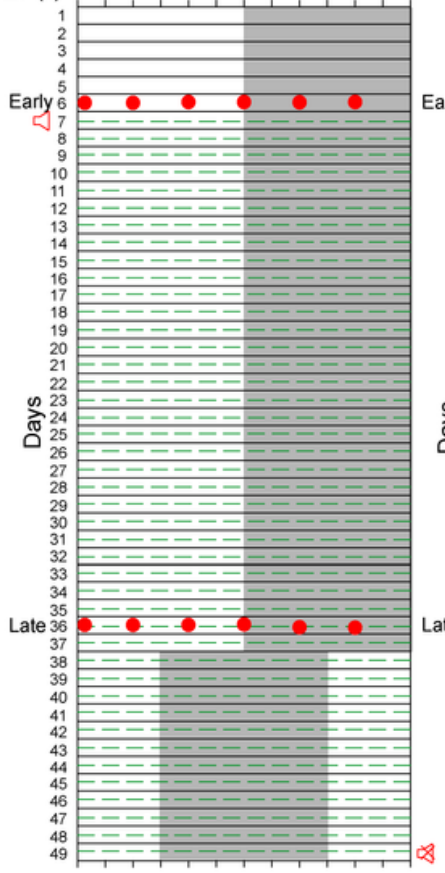

LD regimes

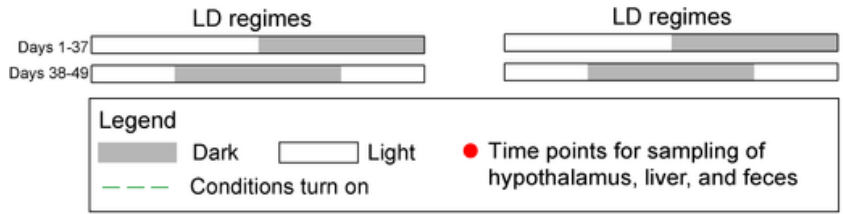

d
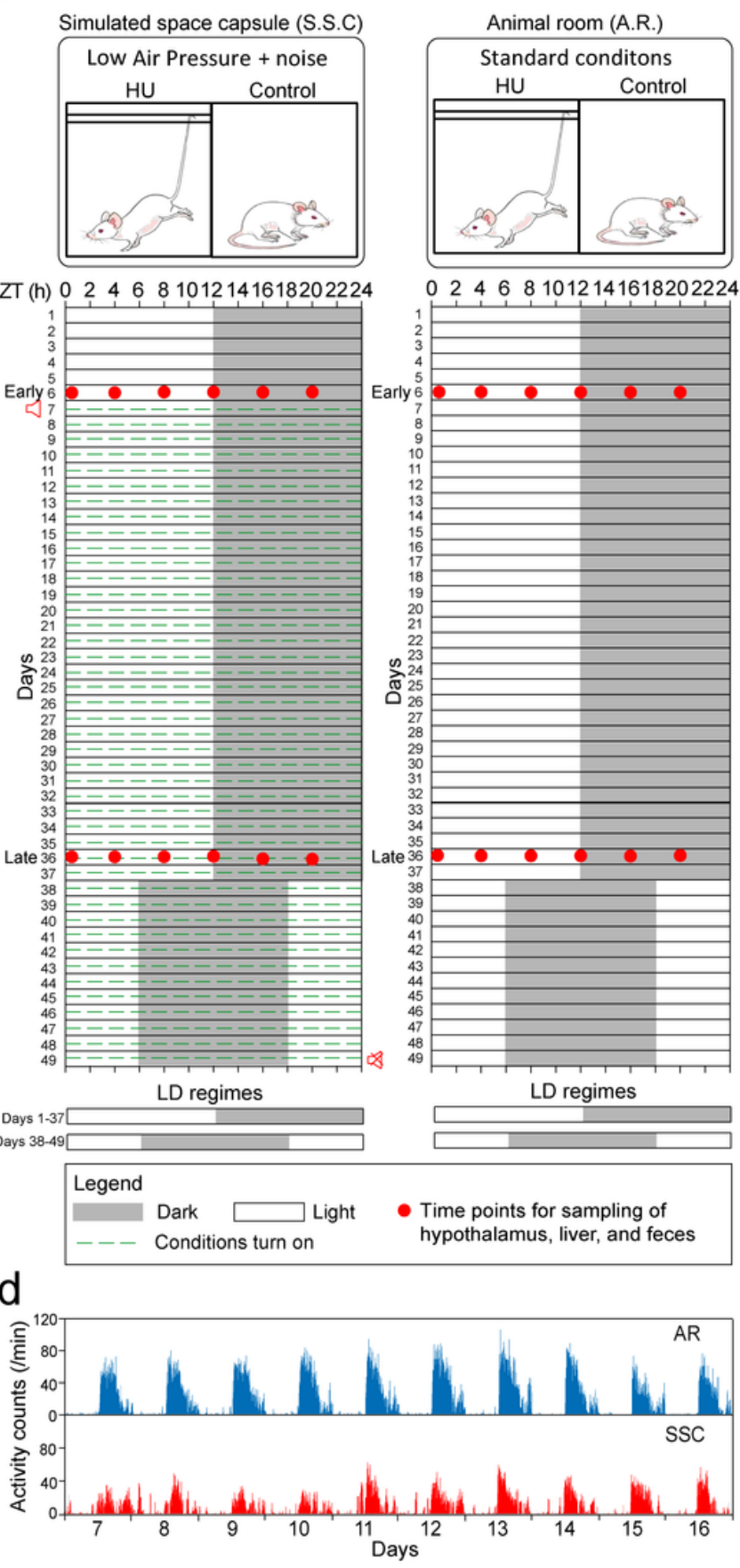

024681012141618202224

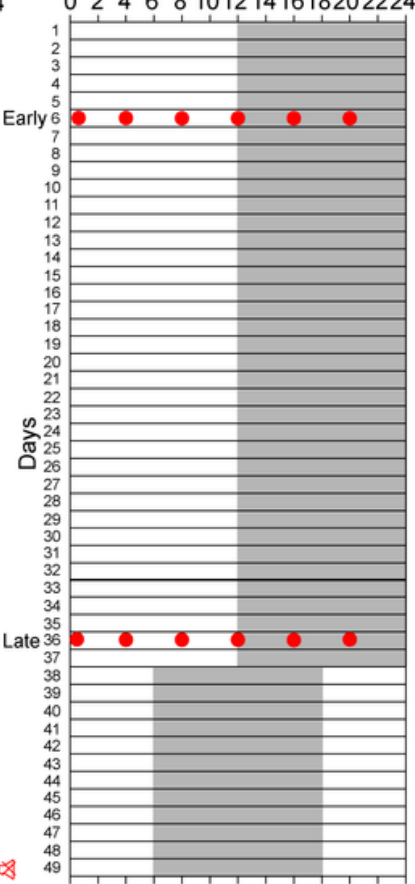

LD regimes b
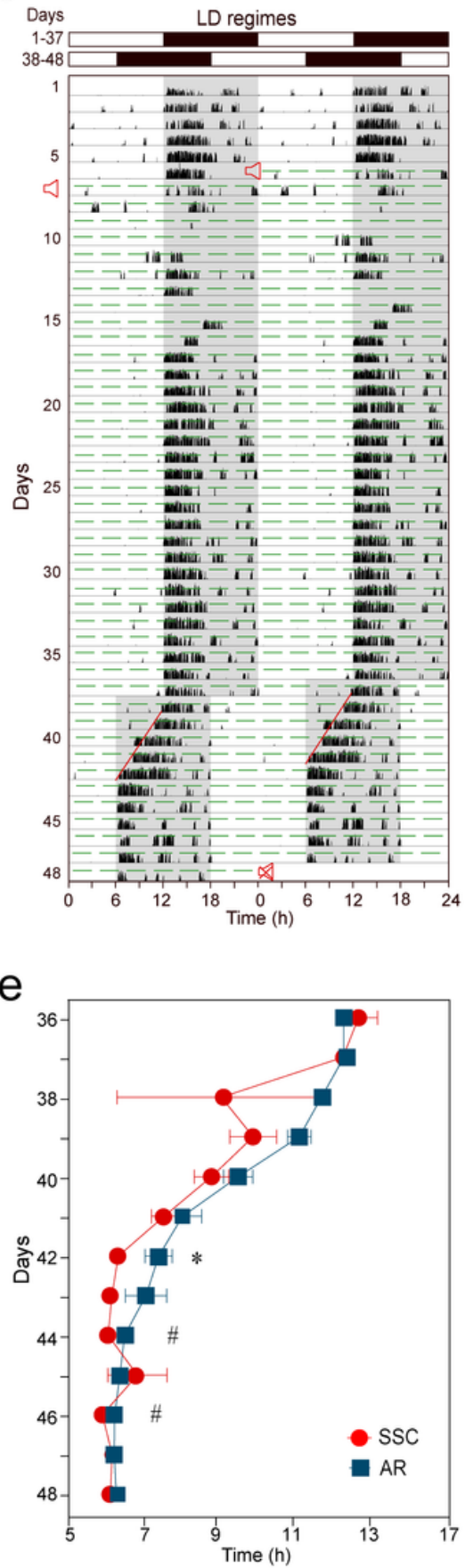

C

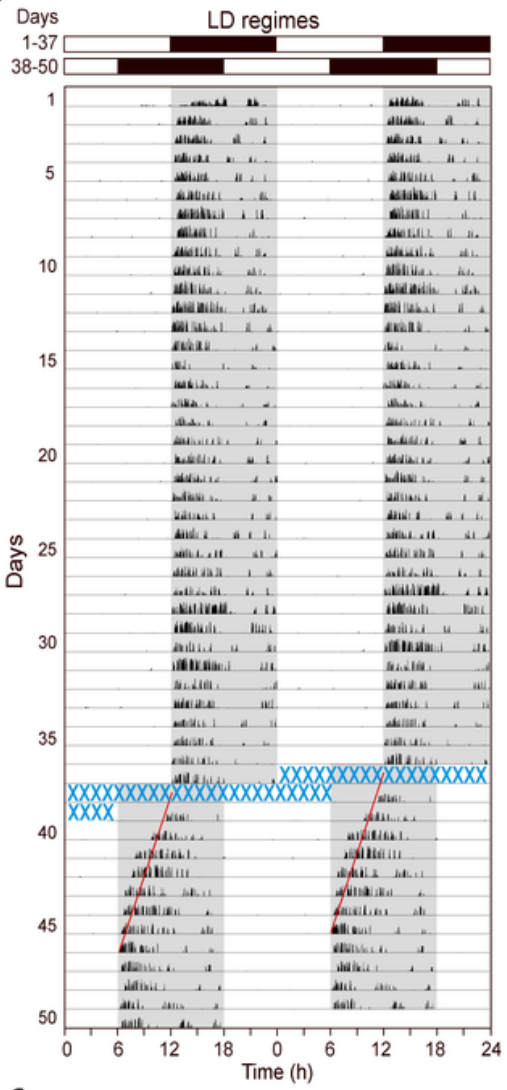

$f_{15}$

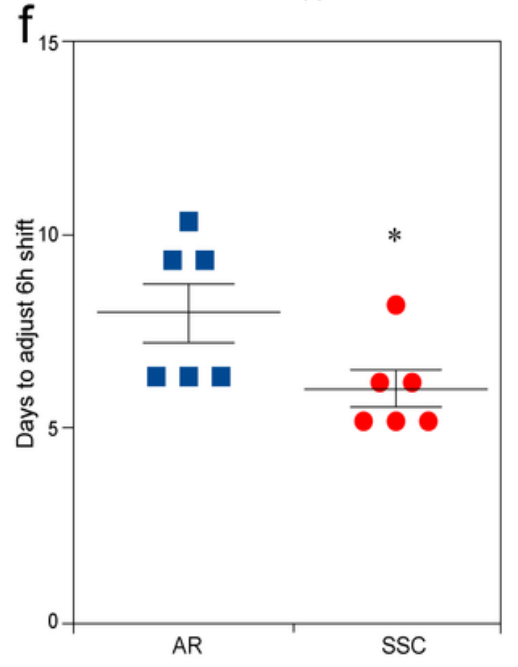

\section{Figure 1}

Experimental schedule and mice running wheel rhythm data. (a) Diagram of the experimental schedule. The experiments in the simulated space capsule and the animal room were carried out simultaneously. The hind limb unloaded (HU) mice or control mice were placed in both simulated space capsule and animal room. The gray squares represent the dark episodes, the white squares represent the light episodes, the green dotted lines indicate that the capsule was being operated and was loaded with conditions of low atmospheric pressure and noise, the red circles represent the time points of sampling of tissues and feces every $4 \mathrm{~h}$. Speakers symbol and speakers symbol with crosses denote the start and end of noise, respectively, simultaneous with exposure to the low atmospheric pressure. $(b, c)$ Representative 
double-plotted actograms of the wheel-running activity of mice in the (b) simulated space capsule (c) and animal room. The mice were subjected to a $6-h$ phase advance in LD cycles from day 38 to the end $(n=6)$. Crosses in blue denote the missing data on days 38-39. (d) The number of activities of mice per minute in the simulated space capsule and the animal room. (e) Daily onset of locomotion in the 6-h phase advance after change in lighting regime $(n=6)$. (f) The days to adjust to the 6 -h phase advance. (d) were calculated and analyzed within days 7-16 of the experiment. One-way ANOVA was used to compare the differences between groups: (e-f) are the mean (SE), ${ }^{\star} p<0.05,{ }^{\star \star} p<0.05, \# p<0.001$. SSC: simulated space capsule; AR: animal room; HU: hind limb unloading; Ctrl: control. 
a
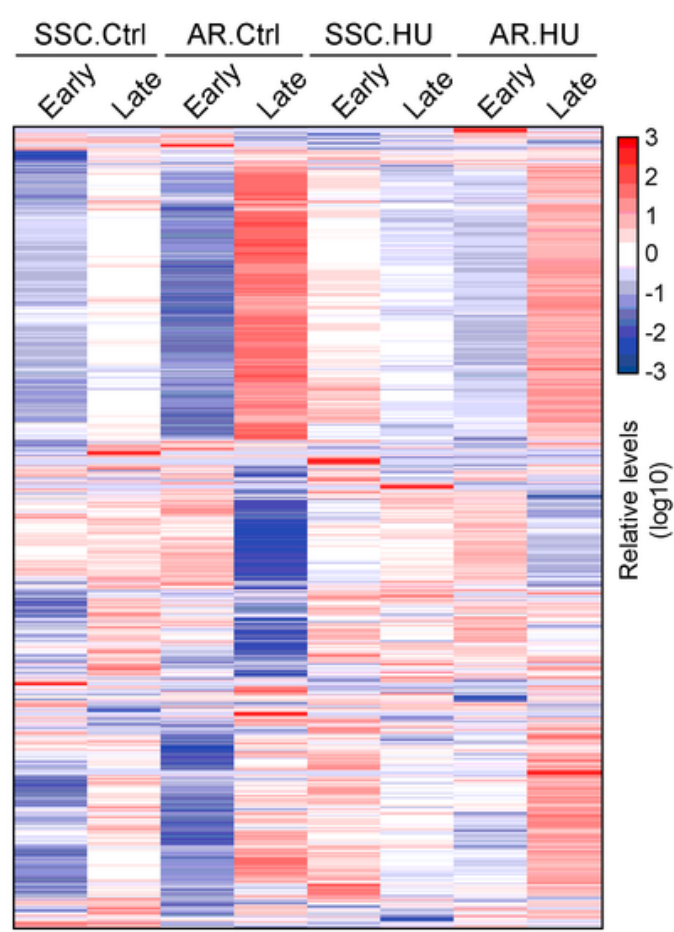

C

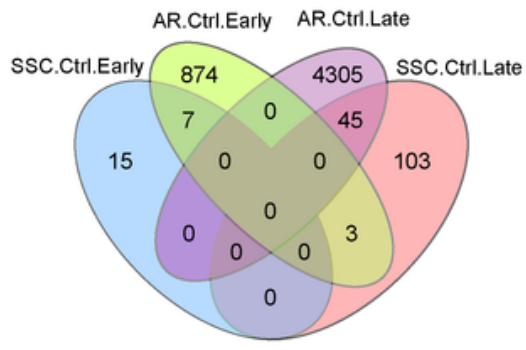

d

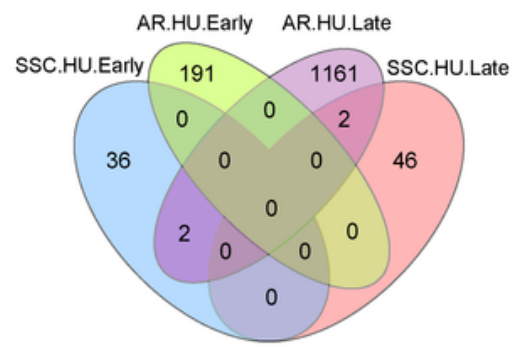

b

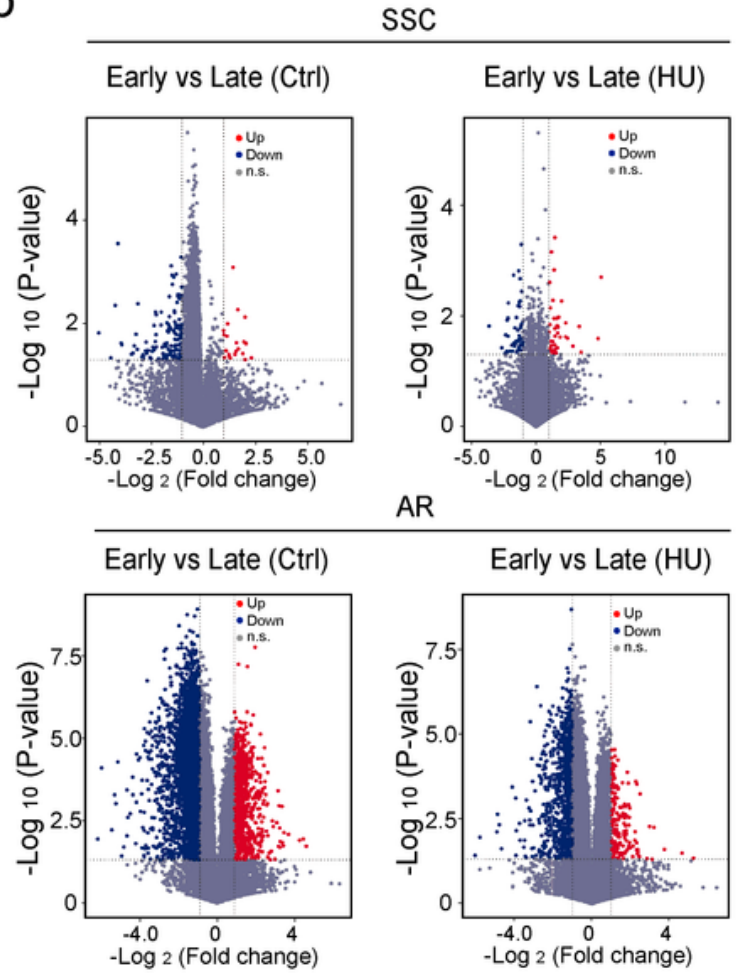

e

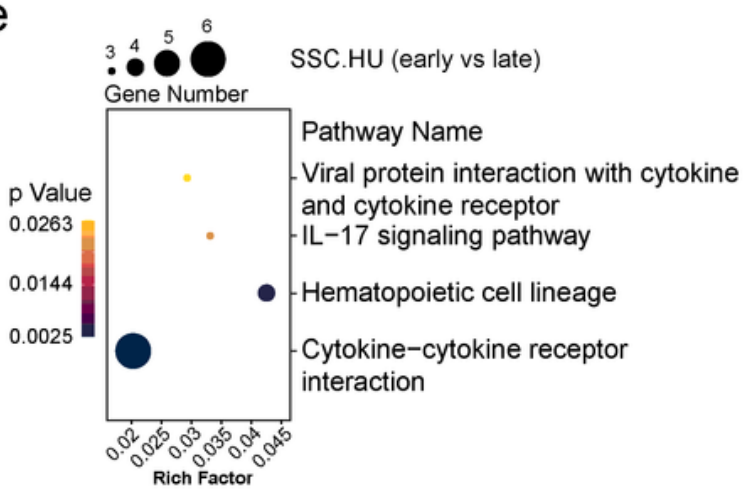

f

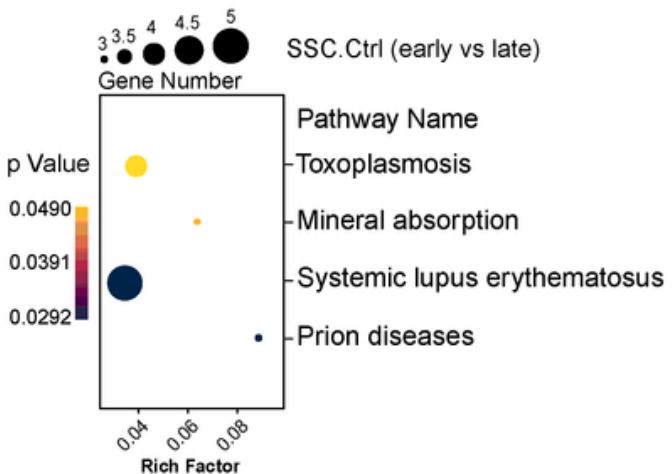

Figure 2

RNA-seq analysis of differentially expressed genes in the hypothalamus. (a) Heat map of genes expressed under different conditions. The values are averages of biological triplicates from independent experiments at six time points. (b) Volcano plot of differentially expressed genes in mouse hypothalamus. (c,d) Venn diagram showing the numbers of DEGs of control mice (c) and HU mice (d). The numbers of DEGs correspond to (b). (e,f) KEGG pathway analyses showing the pathways of the 
DEGs with significant p-values of HU mice between the early and late stages in the capsule (e), and control mice between the early and late stages in the capsule (f). SSC: simulated space capsule; AR: animal room; HU: hind limb unloading; Ctrl: control.

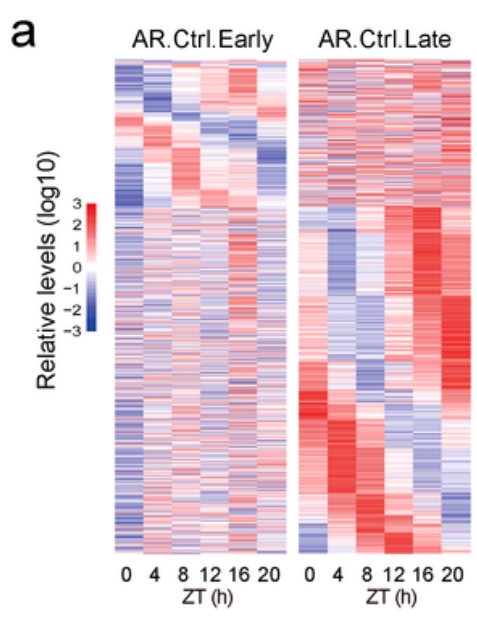

b

AR.HU.Early AR.HU.Late

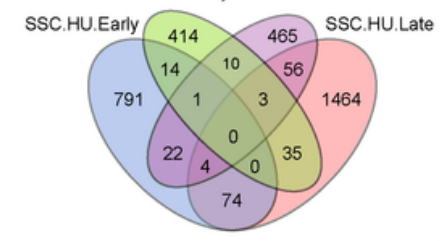

C

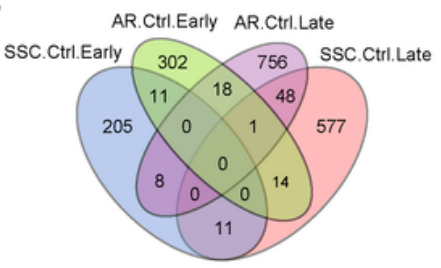

d

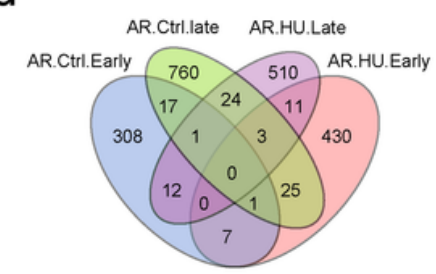

e

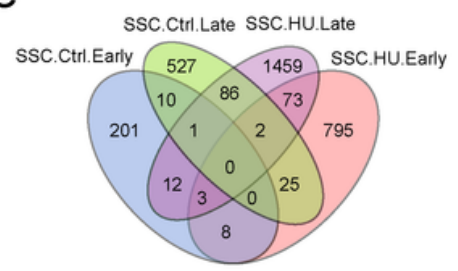

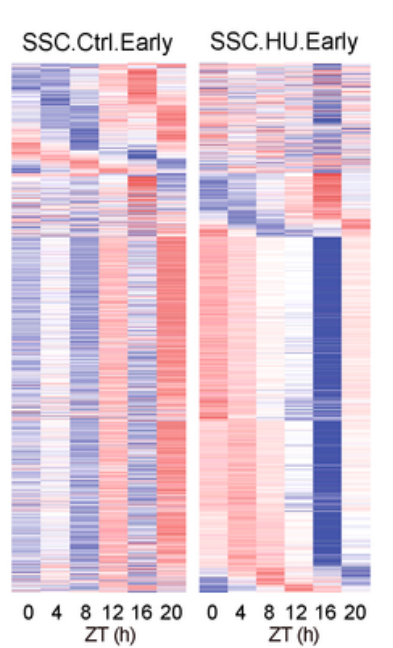
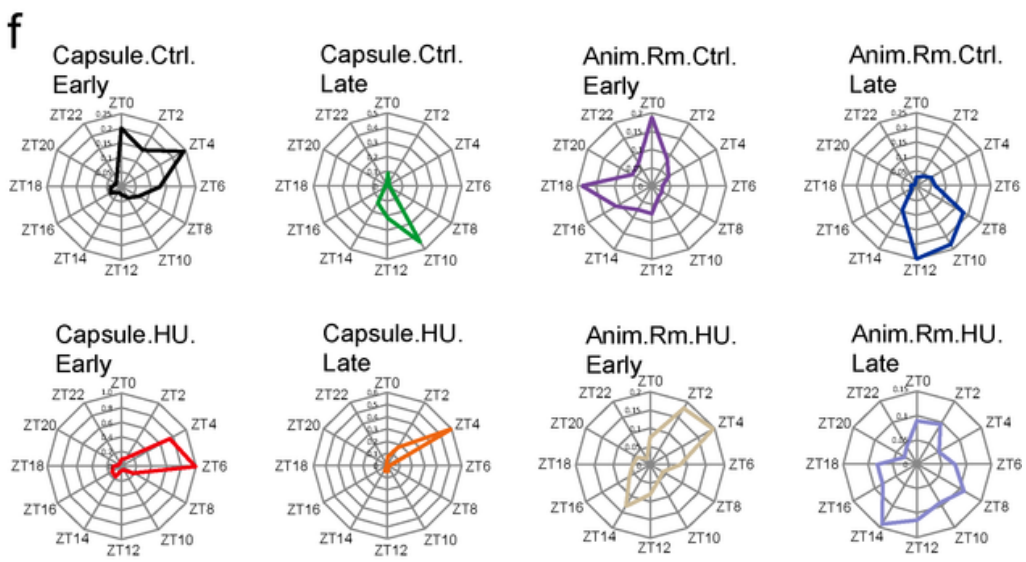

g

Arrhythmic in AR.Ctrl.Early rhythmic in AR. HU. Early
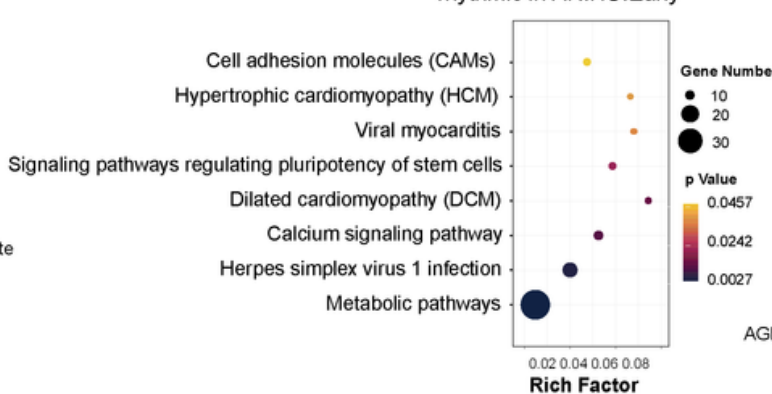

h
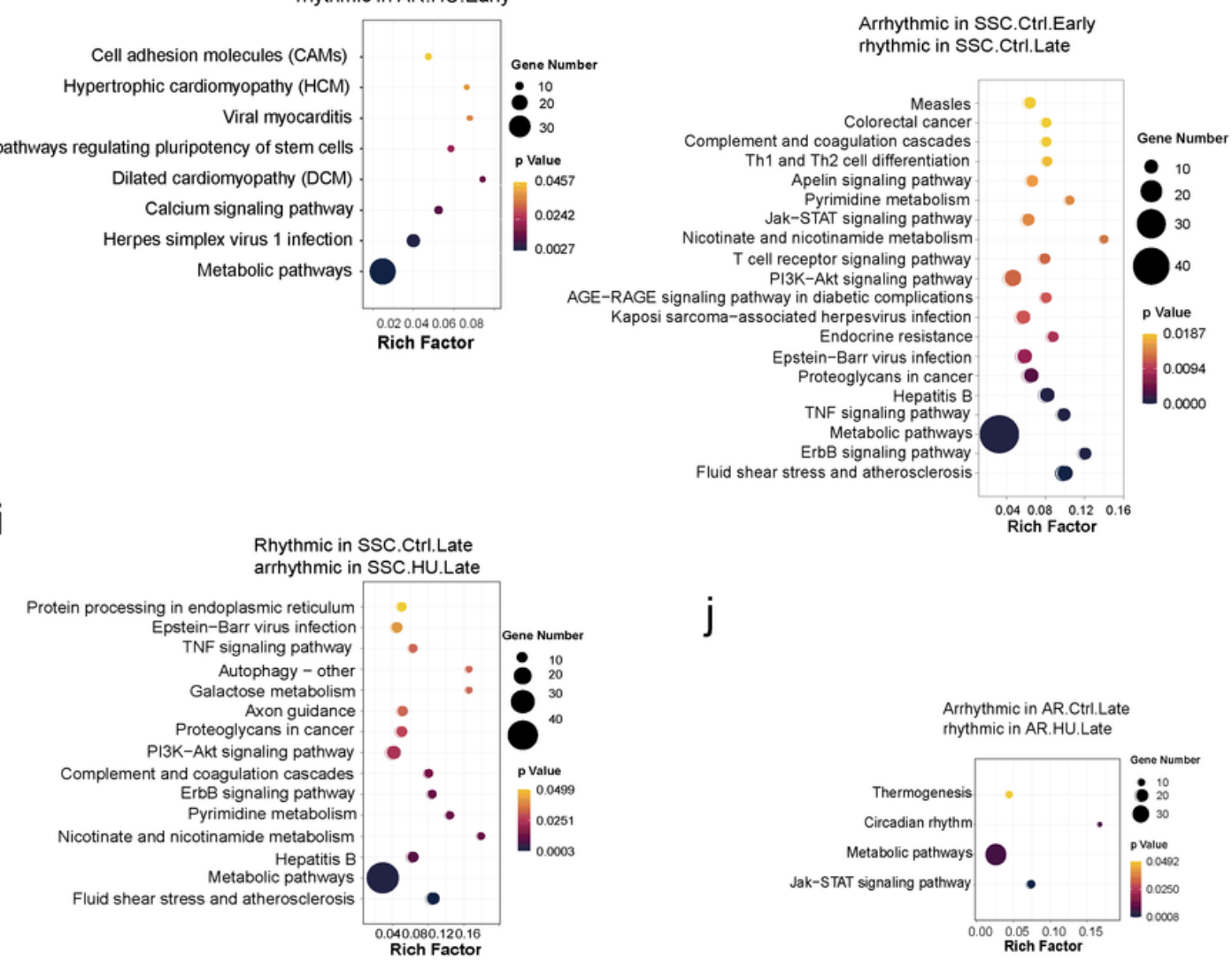

$.040 .08 \quad 0.120 .16$
Rich Factor

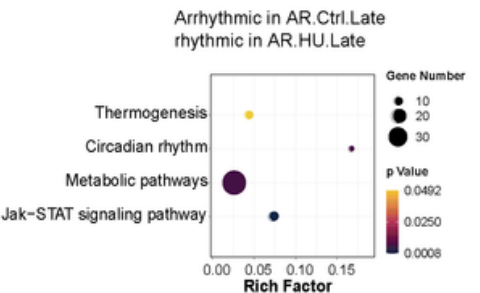

Figure 3

Changes in circadian rhythms at the transcriptomic level. (a) Heat maps of DEGs showing different circadian expression patterns of mice under the indicated conditions. Row normalization was performed and Euclidean distance used to display the hierarchy clustering. (b-e) Venn diagrams of DEG numbers under different conditions. (f) Radar map of circadian phase distribution. (g-j) KEGG pathway analysis of 
the genes showing changes in rhythmicity under different conditions or between different stages. SSC: simulated space capsule; AR: animal room; HU: hind limb unloading; Ctrl: control.
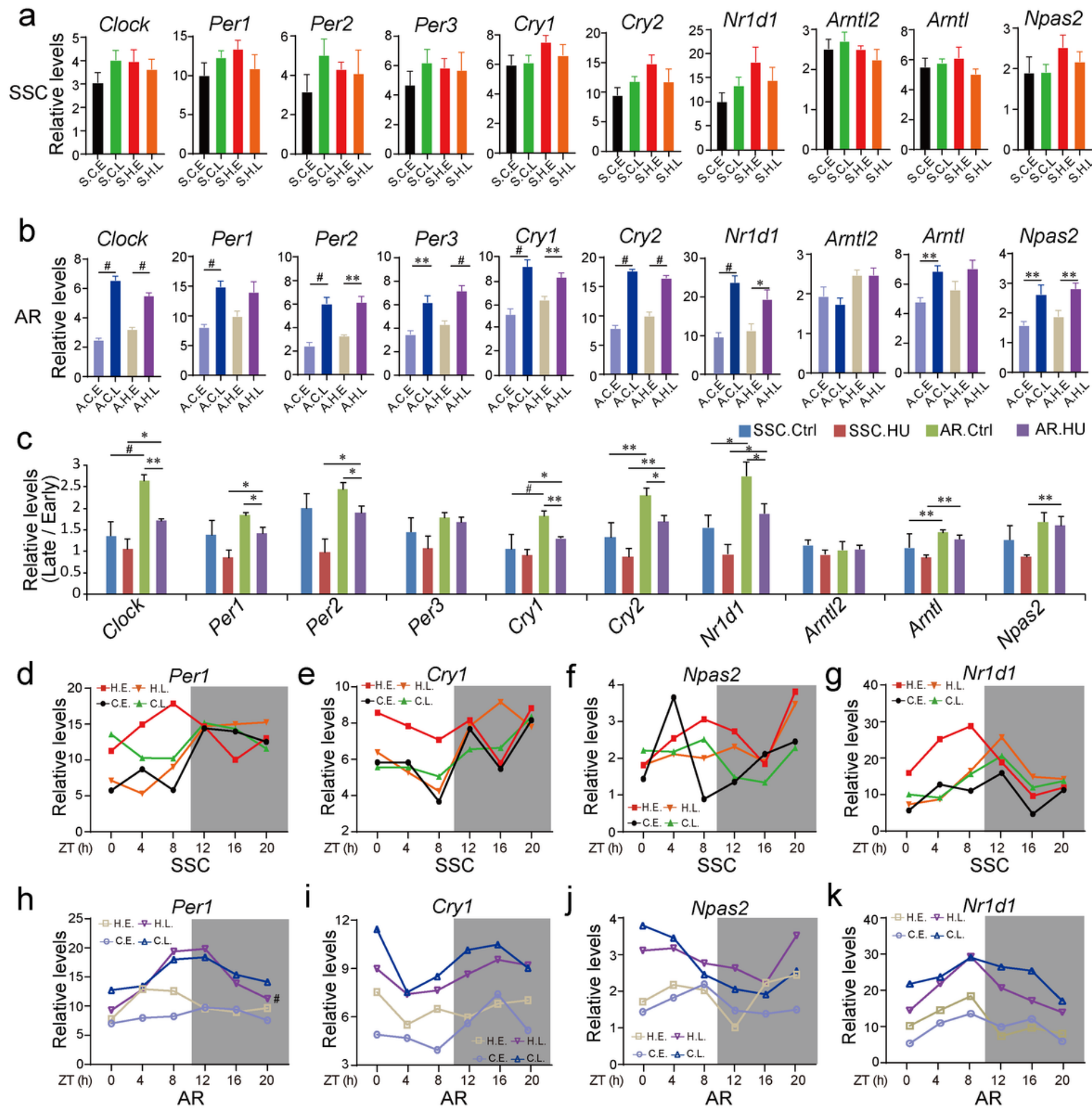

\section{Figure 4}

Changes in circadian clock gene expression in the mouse hypothalamus under different conditions. $(a, b)$ Expression of circadian clock genes in the hypothalamus in the simulated space capsule (a) and the animal room (b). (c) Normalized levels of clock genes. The values are gene expression levels at the late stage relative to those at the early stage. Values are the means of six time points and are presented as 
mean \pm SE. (d-k) Expression of circadian clock genes from ZT 0:00 to 20:00 on the next day in increments of $4 \mathrm{~h}$ in the simulated space capsule (d-g) and the animal room (h-k). The curves are colored the same to those in (a) and (b). (a-c) Significance was tested by One-Way ANOVA. Values are the means of six time points and are presented as mean \pm SE. ${ }^{*} p<0.05,{ }^{*} p<0.01, \# p<0.001$. H.E.: HU mice at the early stage; H.L.: $\mathrm{HU}$ mice at the late stage; C.E.: control mice at the early stage; C.L.: control mice at the late stage.

a

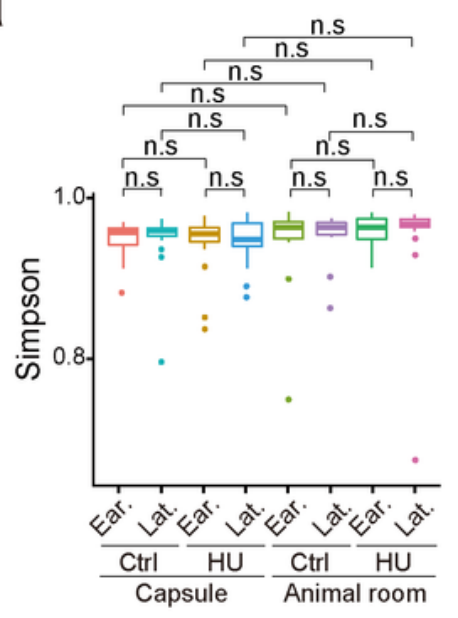

b

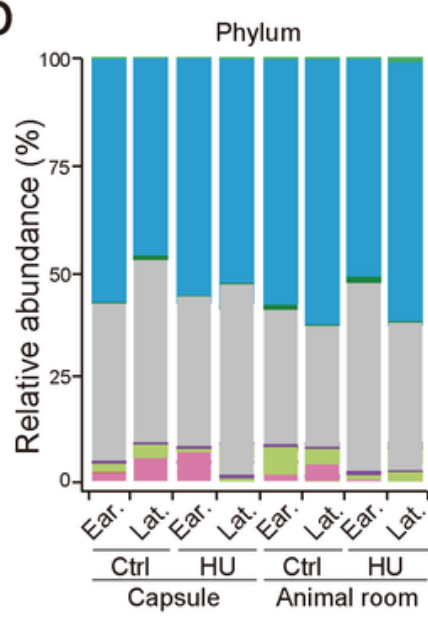

C

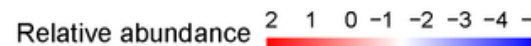
(log10 OUT counts)

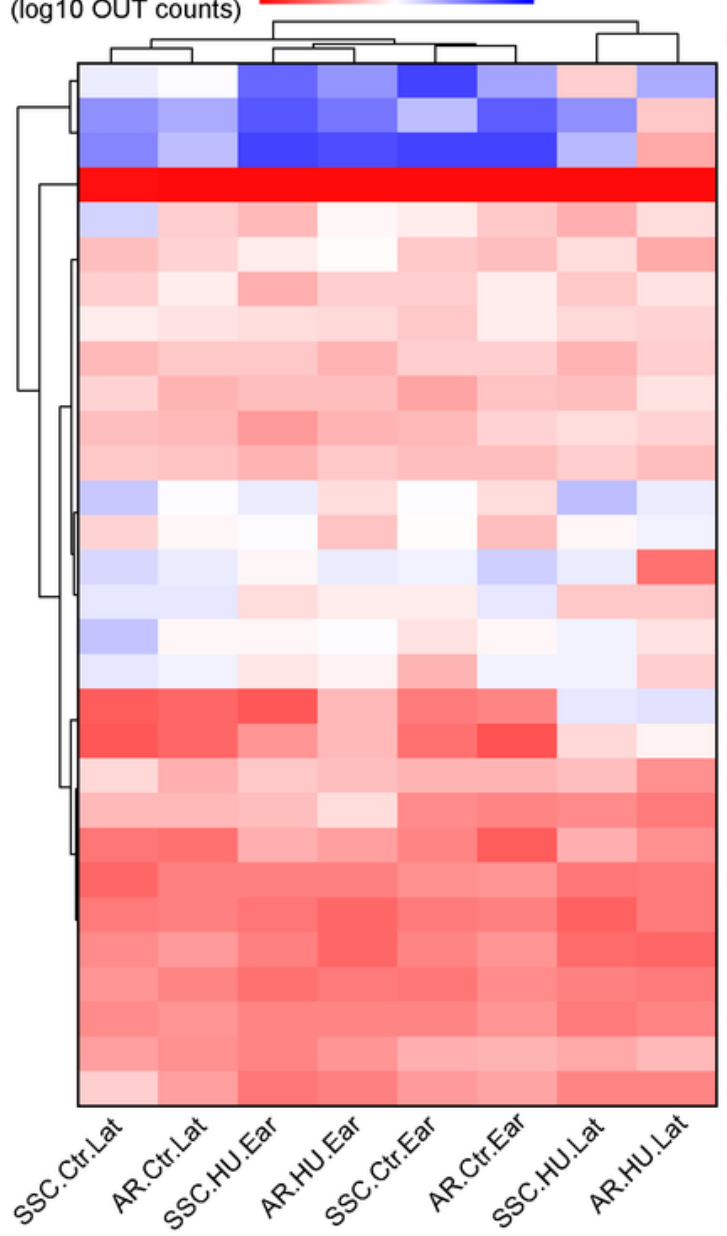

Genus level Anaerostipes Cupriavidus Paraprevotella unclassified Mucispirillum Desulfovibrio Anaerotruncus Dehalobacterium Roseburia Coprobacillus Clostridium Parabacteroides Lactococcus Lupinus Candidatus_Arthromitus Butyricicoccus Anaeroplasma Streptococcus Akkermansia Allobaculum Adlercreutzia Lactobacillus Sutterella Coprococcus Ruminococcus Prevotella

Bacteroides Oscillospira Dorea Odoribacter

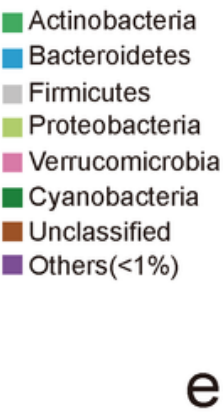

e
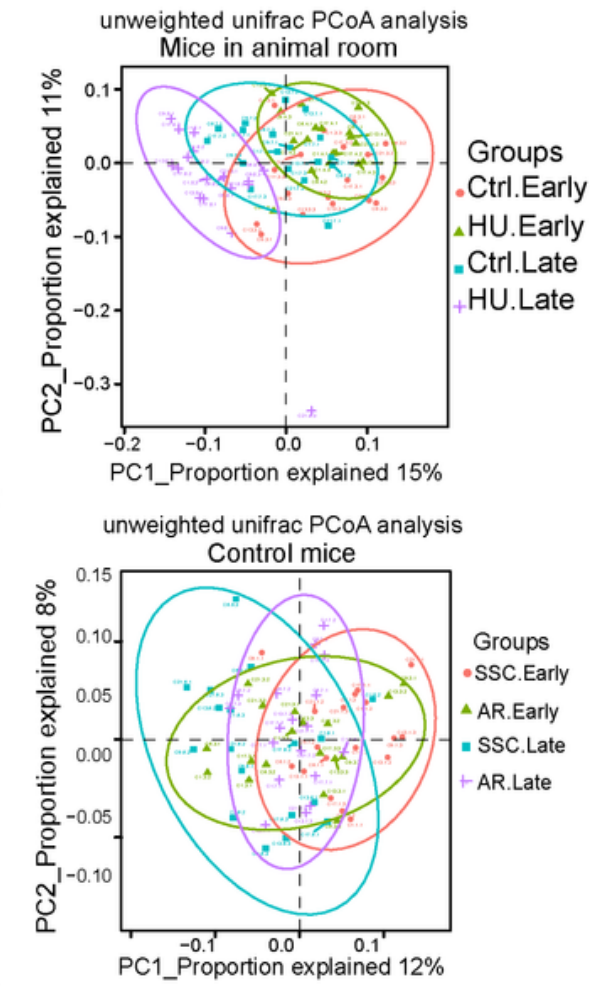

g

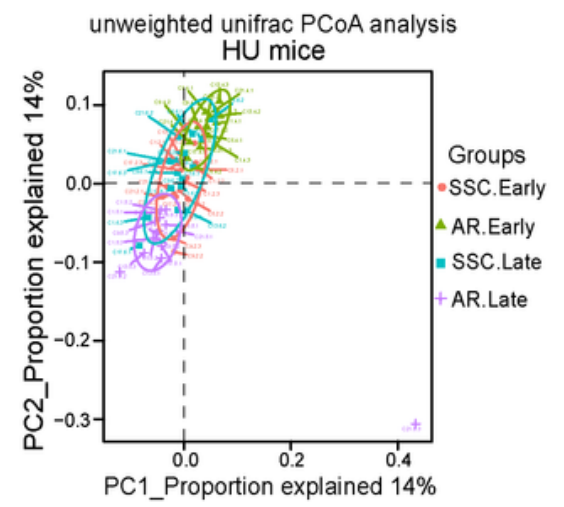


Analysis of the mouse gut microbiome under different conditions. (a) Simpson's diversity index of the microbiome under the indicated conditions. Significance was tested by one-way ANOVA and post hoc comparisons (Fisher's least significant difference Kruskal-Wallis). (b) Relative taxonomic composition of bacteria in the indicated conditions at the order level. The relative abundance of each selected species was $>1 \%$. (c) Heatmap representation of relative bacterial abundance at the genus level. The values are $\log 10$ OUT counts. Euclidean distance was used to display the hierarchy clustering. (d-g) Plots of principal component analysis (PCoA) based on unweighted UniFrac distances under different conditions. SSC: simulated space capsule; AR: animal room; HU: hind limb unloading; Ctrl: control. 
a

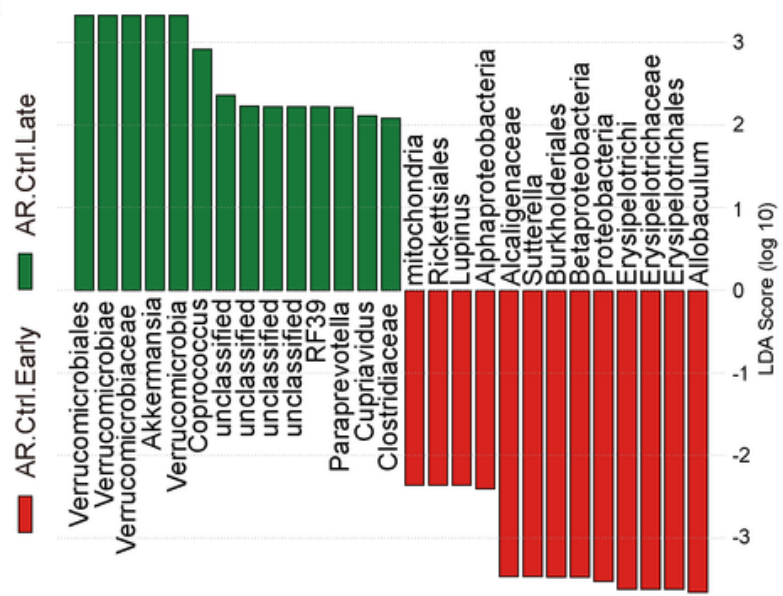

C

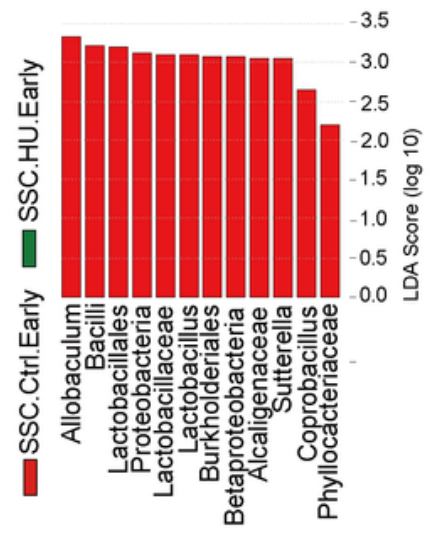

b
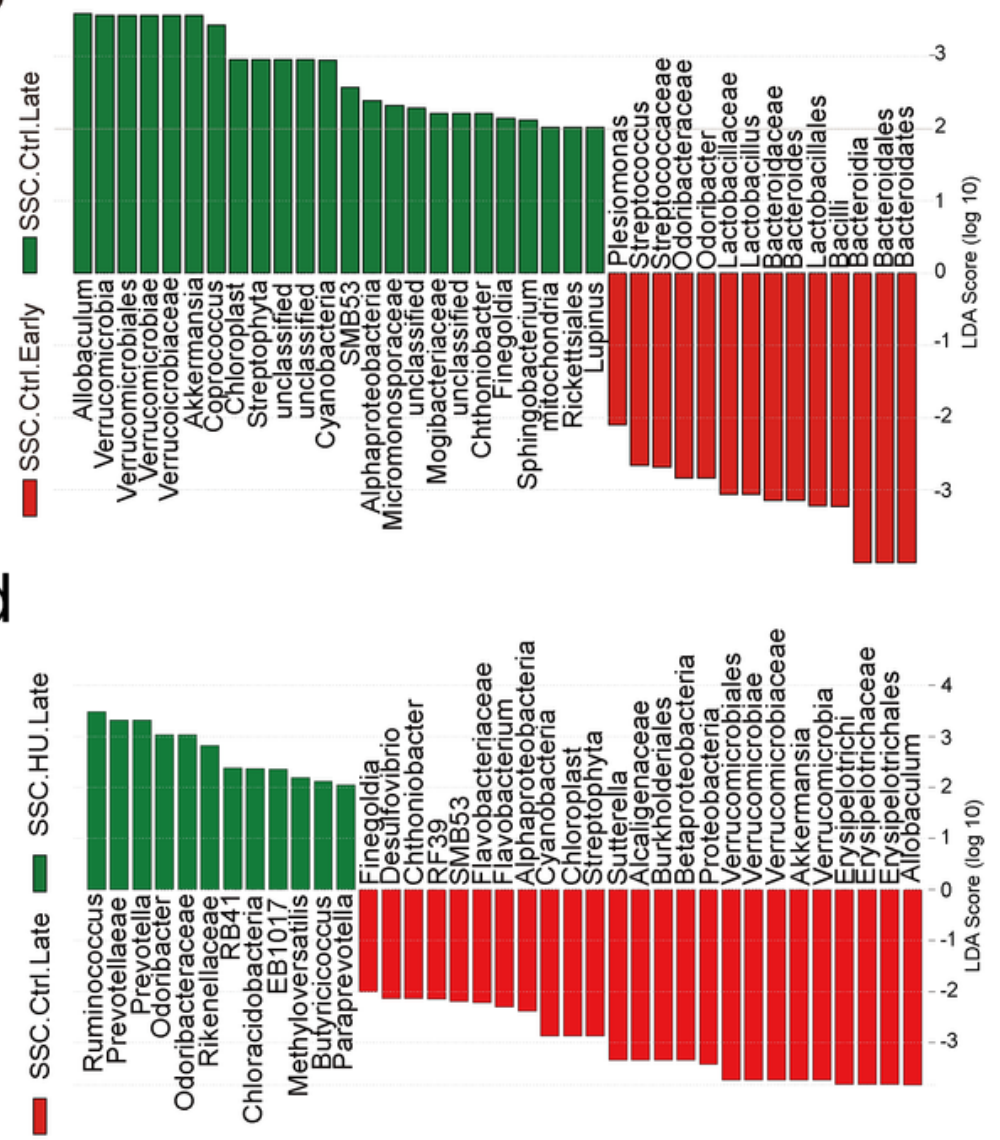

Level 3

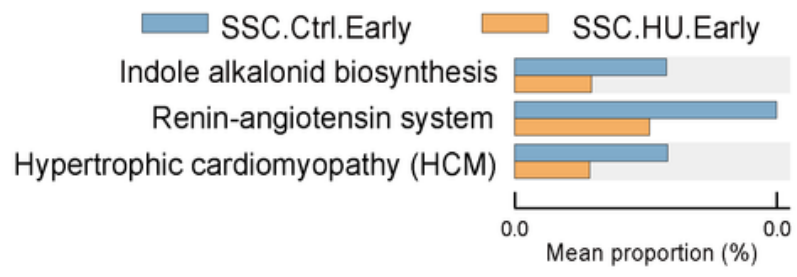

f Level3 Calcium signaling pathway Bacterial invasion of epithelial cells Amyotrophic lateral sclerosis(ALS) Renin-angiotensin system
Hypertrophic cardiomyopathy (HCM) Renin-angiotensin system
Hypertrophic cardiomyopathy (HCM)

SSC.Ctrl. Late Carotenoid biosynthesis Renal cell carcinoma

1

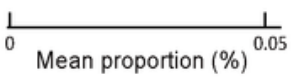

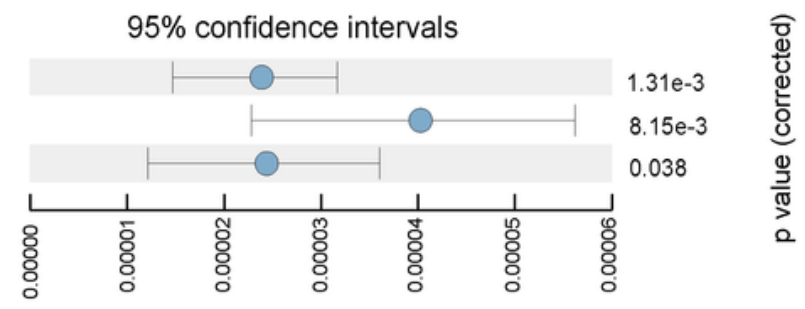

95\% confidence intervals

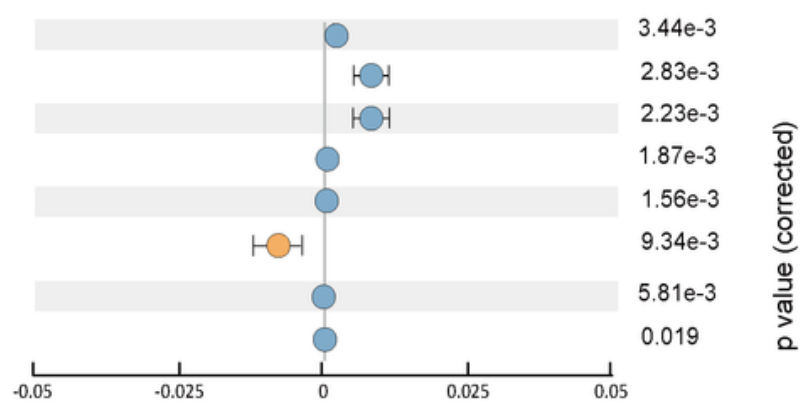

Figure 6

Effects of different conditions on the mouse gut microbiome. (a-d) LDA histogram of bacterial taxa with different abundances under different conditions. (e,f) Predicted KEGG pathways between control and HU mice in the early (e) and late (f) stages. Only some pathways with the lowest p-values are shown, see the full image in supplemental supplementary Figure S7e. The extended error bar plot of significantly 
differential KEGG pathways predicted using PICRUSt. SSC: simulated space capsule; AR: animal room; $\mathrm{HU}$ : hind limb unloading; Ctrl: control.
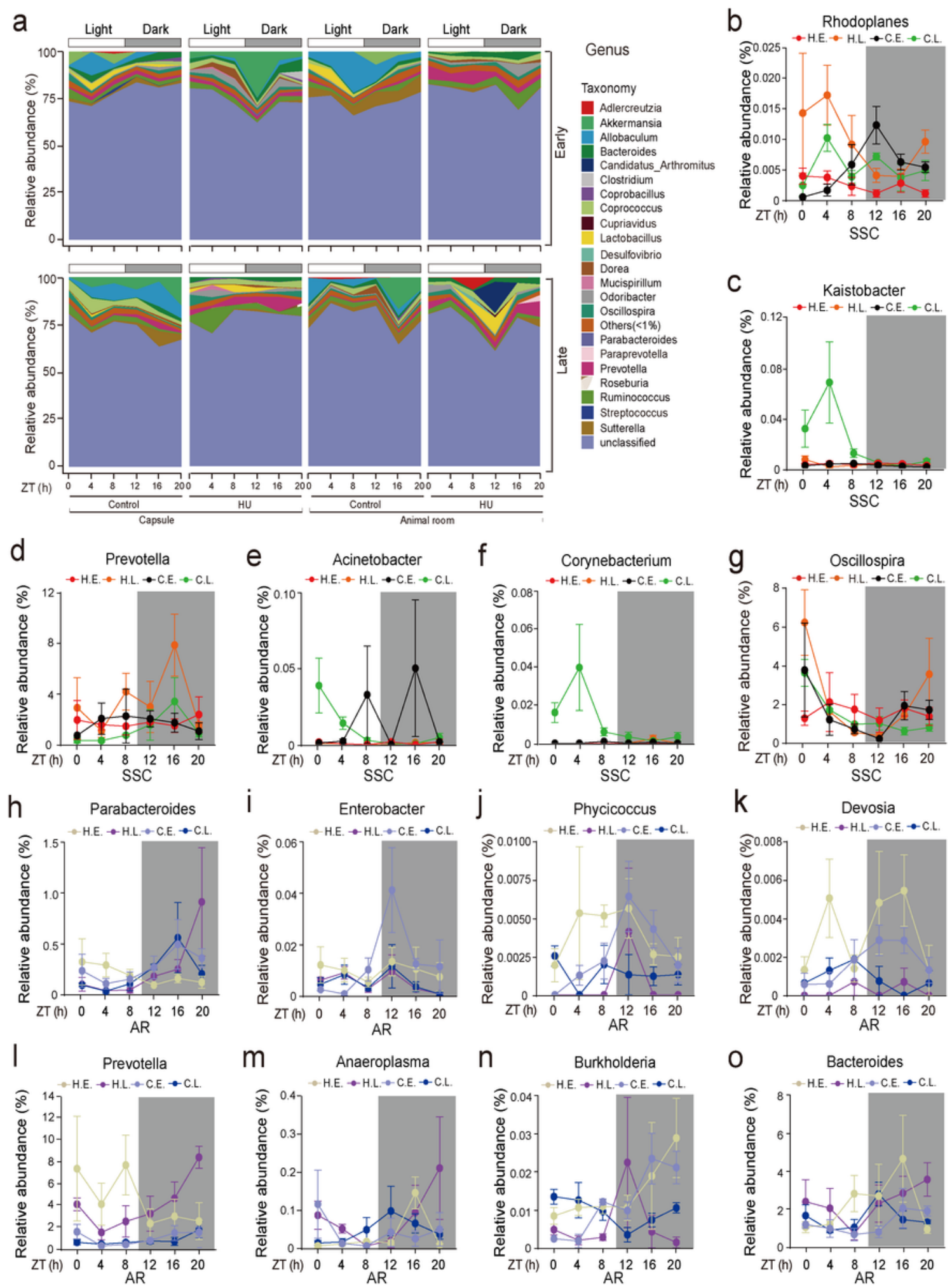

Figure 7

Changes in abundance rhythmicity of the gut microbiome at the genus level. (a) Rhythmic changes in relative abundance at the genus level. Bars in white and grey denotes light and dark periods, respectively. (b-o) Relative abundance of significant taxa in the capsule or the animal room across the time points. 
n=3. H.E.: HU mice at the early stage; H.L.: HU mice at the late stage; C.E.: control mice at the early stage; C.L.: control mice at the late stage.

\section{Supplementary Files}

This is a list of supplementary files associated with this preprint. Click to download.

- Additionalfile1.docx

- Additionalfile2SupplementaryTableS1.xIsx

- Additionalfile3SupplementaryTableS2.xIsx

- Additionalfile4SupplementaryTableS3.xIsx

- Additionalfile5SupplementaryTableS4.xlsx

- Additionalfile6SupplementaryTableS5.xIsx

- Additionalfile7SupplementaryTableS6.xIsx

- Additionalfile8SupplementaryTableS7.xIsx 